\title{
Configuration of Zeros of Isochronous Vector Fields of Degree 5
}

\author{
Julio Cesar Avila $\mathbb{D}^{1},{ }^{1}$ Montserrat Corbera $\mathbb{D}^{2},{ }^{2}$ and Martín Eduardo Frías-Armenta $\mathbb{D}^{3}$ \\ ${ }^{1}$ Tecnologico de Monterrey, Campus Sonora Norte, Blvd. Enrique Mazón López 965, C. P. 83000, Hermosillo, Sonora, Mexico \\ ${ }^{2}$ Departament D'Enginyeries, Universitat de Vic-Universitat Central de Catalunya (UVic-UCC), C. de La Laura 13, 08500 Vic, \\ Barcelona, Spain \\ ${ }^{3}$ Departamento de Matemáticas, Universidad de Sonora, Hermosillo, Mexico
}

Correspondence should be addressed to Julio Cesar Avila; jcavila@tec.mx

Received 17 July 2021; Accepted 31 August 2021; Published 30 November 2021

Academic Editor: Baltazar Aguirre Hernandez

Copyright (c) 2021 Julio Cesar Avila et al. This is an open access article distributed under the Creative Commons Attribution License, which permits unrestricted use, distribution, and reproduction in any medium, provided the original work is properly cited.

In this paper, we give the algebraic conditions that a configuration of 5 points in the plane must satisfy in order to be the configuration of zeros of a polynomial isochronous vector field. We use the obtained results to analyze configurations having some of its zeros satisfying some particular geometric conditions.

\section{Introduction}

We start defining an isochronous vector field, and we express its general associated 1 -form, with its respective residues.

An isochronous vector field $X$ is as a complex polynomial vector field on $\mathbb{C}$ whose zeros are all isochronous centers. A center is isochronous if the periods of the trajectories surrounding it are constant.

Let $X$ be a complex polynomial vector field on $\mathbb{C}$ of degree $n \geq 1$, nonidentically zero, as follows:

$$
X=\left(b_{n} z^{n}+b_{n-1} z^{n-1}+\cdots+b_{1} z+b_{0}\right) \frac{\partial}{\partial z}=\frac{1}{\lambda}\left(z-p_{1}\right) \cdots\left(z-p_{n}\right) \frac{\partial}{\partial z}
$$

where the coefficients can be calculated by Vieta's formulas, in particular $\lambda=1 / b_{n}$. An isochronous vector field $X$ is characterized by their associated 1 -form:

$$
\eta=\frac{\mathrm{d} z}{b_{n} z^{n}+b_{n-1} z^{n-1}+\cdots+b_{1} z+b_{0}}=\frac{\lambda \mathrm{d} z}{\left(z-p_{1}\right) \cdots\left(z-p_{n}\right)},
$$

which has a unique zero at infinity of multiplicity $n-2$ and simple poles with nonzero pure imaginary residues. For $n \geq 2$, the residue of $\eta$ at $p_{j}$ is

$$
r_{j}=\frac{\lambda}{\left(p_{j}-p_{1}\right) \cdots\left(\widehat{p_{j}-p_{j}}\right) \cdots\left(p_{j}-p_{n}\right)},
$$

where the hat $\left(\widehat{p_{j}-p_{j}}\right)$ means that the factor $\left(p_{j}-p_{j}\right)$ is omitted (see [1]).

The following well-known result characterizes the polynomial isochronous vector fields.

Theorem 1 (see $[1,2])$. Let $X$ be a complex polynomial vector field on $\mathbb{C}$ of degree $n \geq 2$ defined as in (1); then, the following statements are equivalent: 


$$
X^{\prime}\left(p_{j}\right)=\frac{1}{\lambda}\left(p_{j}-p_{1}\right) \cdots\left(\widehat{p_{j}-p_{j}}\right) \cdots\left(p_{j}-p_{n}\right) \in i \mathbb{R}^{*}, \quad \text { for all } j=1, \ldots, n \text {. }
$$

(c) Their residues satisfy

$$
r_{j} \in i \mathbb{R}^{*}, \quad \text { for all } j=1, \ldots, n \text {. }
$$

Here, $i \mathbb{R}^{*}$ is the set of the pure imaginary complex numbers different from zero.

Next, we characterize the polynomial isochronous vector fields over the Riemann spheres in terms of the quotients of its residues.
Definition 1. We say that the collection of zeros $\left[p_{1}, \ldots, p_{n}\right]$ is an isochronous configuration if there exists a rotation $e^{i \theta_{0}}$ such that the vector field $e^{i \theta_{0}} X$ is isochronous.

If the residues $\left[r_{1}, \ldots, r_{n}\right]$ belong to the line $z=\rho e^{i \theta_{0}}$ for some $\theta_{0}$, then the configuration $\left[p_{1}, \ldots, p_{n}\right]$ is isochronous. In short, the configuration $\left[p_{1}, \ldots, p_{n}\right]$ is isochronous if and only if

$$
\frac{r_{j}}{r_{k}}=-\frac{\left(p_{k}-p_{1}\right)\left(p_{k}-p_{2}\right) \cdots\left(\widehat{p_{k}-p_{k}}\right) \cdots\left(\widehat{p_{k}-p_{j}}\right) \cdots\left(p_{k}-p_{n}\right)}{\left(p_{j}-p_{1}\right)\left(p_{j}-p_{2}\right) \cdots\left(\widehat{p_{j}-p_{j}}\right) \cdots\left(\widehat{p_{j}-p_{k}}\right) \cdots\left(p_{j}-p_{n}\right)} \in \mathbb{R}^{*},
$$

for all $j$ and $k=1, \ldots, n$ with $j \neq k$. Here, $\mathbb{R}^{*}$ is the set of real numbers different from zero.

We can associate to each isochronous vector field $X$ a weighted $n$-tree in the following way. The $n$ vertices correspond to the $n$ zeros of $X$, and two vertices are connected with an edge if the basins of the corresponding centers are adjacent and the weights are the periods $[1,3]$. We know that each embedded $n$-tree (without weights) is realized by an isochronous vector field $X$ and that if the phase portraits of two different isochronous vector fields are topologically equivalent, then they have the same embedded $n$-tree (see [3]).

A topological classification of the isochronous vector fields of degree 2 can be found in [1, 4-6]. In [1], the authors characterize the isochronous vector fields of degree $n$ in terms of the shape of the configuration of zeros when $n=3$ and 4 , and they give partial results for $n \geq 5$ when the zeros present some symmetries. The known results for $n=4$ and $n=5$ are summarized in the next section. Another reference about polynomial vector fields is given in [7] and rational vector field on the Riemann sphere in [8]. Finally, more general vector fields are studied in $[9,10]$.

The aim of this paper is to characterize the isochronous vector fields of degree 5 in terms of the configurations of zeroes without imposing any condition of symmetry.

If a polynomial vector field $X$ over the Riemann sphere is given, we can change the coordinates in such a way that $X$ has a zero at 0 and another zero at 1 because an affine transformation over the Riemann space does not change the main characteristics of a polynomial vector field, in particular the condition of to be isochronous, $[1,3]$. Then, if the complex polynomial vector field $X$ has degree 5 , it has 8 free real parameters: six for the zeros and two for the main coefficient. Assuming that the position of two zeros is fixed, it will be proved that there can be up to seven different threeparameter families of isochronous configurations, see Theorem 6. Notice that the family of 5-degree isochronous vector fields has only three real parameters. There are still too much parameters to give a result providing all the possible shapes of the zeros of the isochronous configurations for $n=5$. Nevertheless, we can reduce the parameter space by fixing either the position or the shape of some zeros. At the end of the paper, we will analyze the shape of the isochronous configurations in some particular cases where the zeros present some symmetries and we will complete some of the cases studied in [1].

The paper is structured as follows. In the next section, we give a summary of the known results on isochronous vector fields of degree 5. After that, in the following section, we describe how to solve the set of equation (6) for an arbitrary vector field of degree 5. Finally, in the last section, we give explicitly the isochronous configurations in some particular cases: when four zeros are at the vertices of either a parallelogram or an isosceles trapezoid, when two zeros are on the line orthogonal to the line passing through other two zeros, and when three zeros are at the vertices of an equilateral triangle. We give numerical examples of some isochronous configurations with three zeros at the vertices of two isosceles triangles when $p_{1}=0, p_{2}=1$, and $p_{3}=(1 / 2)+$ $i(1 / 25)$ and when $p_{1}=0, p_{2}=1$, and $p_{3}=(1 / 2)+i(9 / 10)$. Finally, we give examples of isochronous configurations where the zeros do not satisfy any symmetry. We also analyze the phase portrait of the isochronous vector fields associated to the configurations. In particular, we find either the star or fork topology (Figure 1) in all cases except when 
we have all the zeros in the line. We have not found examples of the line topology when the zeros are not all in a line.

\section{Some Known Results}

In this section, we summarize some known results on isochronous configurations of vector fields of degree $n \geq 4$ given in [1].

Theorem 2 (see [1]). A polynomial vector field $X$ of degree 4 is isochronous if and only if the zeros $p_{1}, p_{2}, p_{3}$, and $p_{4}$ are either in a line or three are in the vertices of a triangle and one is at its orthocenter.

The phase portrait of the isochronous vector fields of degree 5 can have as many different topologies as 5 -trees, so they can have three different topologies (see Figure 1), namely, the star topology, the fork topology, and the line topology.

The next theorem summarizes the results of isochronous configurations that are valid for vector fields of degree $n \geq 5$.

Theorem 3 (see [1]). The following statements hold.

(a) For each $n \geq 3$, if the zeros $p_{1}, p_{2}, \ldots, p_{n}$ are in a line, then $X$ is isochronous and his phase portrait has the line topology

(b) For each $n \geq 4$, if the zeros $p_{2}, p_{3}, \ldots, p_{n}$ are at the vertices of a regular polygon and $p_{1}$ is at its center, then $X$ is isochronous and his phase portrait has the star topology

(c) For each $n \geq 4$, there exist isochronous vector fields with the zeros $p_{1}, \ldots, p_{n-2}$ in a line and the zeros $p_{n-1}$ and $p_{n}$ in new line orthogonal to the previous one In addition, for $n=5$, the following statements hold.

(d) If $p_{1}=(1 / 2), p_{2}=(-1 / 2), p_{3}=i y_{3}, p_{4}=i y_{4}$, and $p_{5}=i y_{5}$, then $X$ is isochronous if and only if $y_{3}+y_{4}+y_{5}=4 y_{3} y_{4} y_{5}$

(e) For $n=5$, if $p_{2}, \ldots, p_{5}$ are at the vertices of a rhombus and $p_{1}$ is at its center and if the residues of $X$ satisfy $r_{1}=-2\left(r_{2}+r_{3}\right), r_{2}=r_{4}$, and $r_{3}=r_{5}$, then $X$ is isochronous; moreover, its phase portrait has the star topology

(f) Assume that $X$ is isochronous and $p_{3}, p_{4}$, and $p_{5}$ are in the bisector line of the segment with endpoints $p_{1}$ and $p_{2}$

(i) If $p_{4}$ is in the convex hull of $p_{1}, p_{2}, p_{3}$, and $p_{5}$ and the residues of $X$ satisfy $r_{4}=r_{1}+r_{2}+r_{3}+r_{5}$ and $\left|r_{1}\right|=\left|r_{2}\right|$, then $X$ is isochronous; moreover, the phase portrait of $X$ has the star topology

(ii) If $p_{3}$ and $p_{4}$ are in the convex hull of $p_{1}, p_{2}$, and $p_{5}$ and the residues of $X$ satisfy $\left|r_{3}\right|<\left|r_{1}\right|+\left|r_{2}\right|+$ $\left|r_{4}\right|$ and $\left|r_{4}\right|<\left|r_{5}\right|$, then $X$ is isochronous; moreover, its phase portrait has the fork topology

Although we are not interested in physical applications in this paper, we mention some of them studied in [11]. For example, let $F(z)=\sum_{j=1}^{n}\left(m_{j} /\left(z-z_{j}\right)\right)$; then, the $j$ th terms may be regarded as the force with which a fixed mass (or electric charge) $m_{j}$ at $z_{j}$ repeals (attracts if $m_{j}<0$ ) a movable unit mass (or charge) at $z$, being the law of repulsion, the inverse distance law. Equivalent interpretation can be made in terms of masses (or charges) repelling according to the inverse-square laws. Actually, in Theorem (3.1) in [10], it says that the zeros of $F(z)$ with all $m_{j}$ real are the points of equilibrium in the field of force due to the systems of $p$ masses (point charges) $m_{j}$ at the fixed points $z_{j}$ repelling a movable unit mass at $z_{j}$ according to the inverse distance law. Note that the expression $F(z)$ has the same form as the 1 -form $\eta$ given in (2) associated to an isochronous vector field $X$, and it can be written as $\eta=\sum_{j=1}^{n}\left(r_{j} /\left(z-p_{j}\right)\right) \mathrm{d} z$, with $r_{j}$ given in (3).

Still another interpretation in $F(z)$ is that each term $m_{j} /\left(z-z_{j}\right)$ is the vector velocity in a two-dimensional flow of an incompressible fluid due to a source of strength $m_{j}$ at $z_{j}\left(\right.$ sink if $\left.m_{j}<0\right)$.

\section{Characterization of the Isochronous Vector Fields of Degree 5}

As previously said, without loss of generality, we can consider that the position of two of the zeros of $X$ are fixed to 0 and 1 .

Assume that $p_{1}=0$ and $p_{2}=1$, and let $p_{3}=x_{3}+i y_{3}$, $p_{4}=x_{4}+i y_{4}$, and $p_{5}=x_{5}+i y_{5}$. From (6), the configuration $\left[p_{1}, \ldots, p_{5}\right]$ is isochronous if and only if $\left(r_{j} / r_{k}\right) \in \mathbb{R}^{*}$ for all $j$ and $k=1, \ldots, n$ with $j \neq k$. Note that we only are interested in configurations with $p_{i} \neq p_{j}$ for $i \neq j$. We define

$$
\begin{aligned}
& e_{1}=\operatorname{Im}\left(\frac{r_{1}}{r_{2}}\right), \\
& e_{2}=\operatorname{Im}\left(\frac{r_{1}}{r_{3}}\right), \\
& e_{3}=\operatorname{Im}\left(\frac{r_{1}}{r_{4}}\right), \\
& e_{4}=\operatorname{Im}\left(\frac{r_{1}}{r_{5}}\right), \\
& e_{5}=\operatorname{Im}\left(\frac{r_{2}}{r_{3}}\right), \\
& e_{6}=\operatorname{Im}\left(\frac{r_{2}}{r_{4}}\right), \\
& e_{7}=\operatorname{Im}\left(\frac{r_{2}}{r_{5}}\right), \\
& e_{8}=\operatorname{Im}\left(\frac{r_{3}}{r_{4}}\right), \\
& e_{9}=\operatorname{Im}\left(\frac{r_{3}}{r_{5}}\right), \\
& e_{10}=\operatorname{Im}\left(\frac{r_{4}}{r_{5}}\right),
\end{aligned}
$$




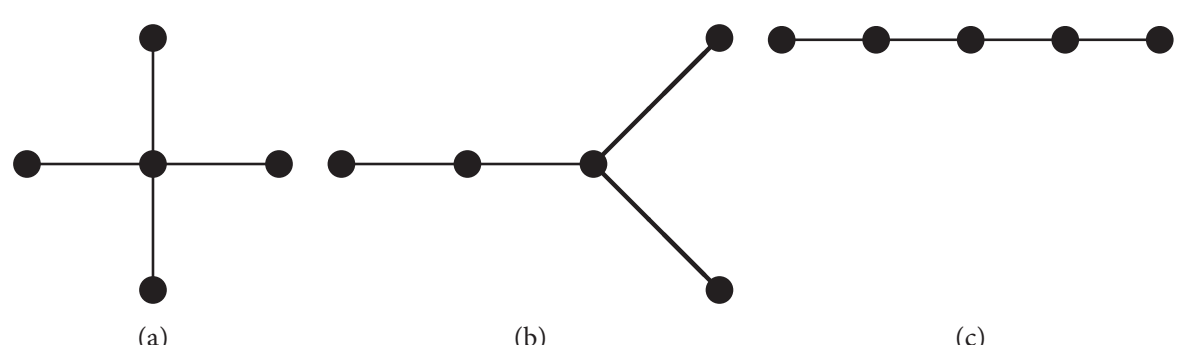

(a)

(b)

(c)

Figure 1: The planar 5-trees. (a) The star topology. (b) The fork topology. (c) The line topology.

The denominators of the functions $e_{i}$ are defined when $p_{i} \neq p_{j}$ for $i \neq j$ so we can drop them. Let $f_{i}$ be the numerator of the factorization of $e_{i}$ for all $i=1, \ldots, 10$. Then, $\left[p_{1}, \ldots, p_{5}\right]$ is isochronous if and only if $\left(x_{3}, y_{3}, x_{4}, y_{4}, x_{5}, y_{5}\right)$ is a solution of the set of polynomial equations:

$$
f_{i}=0, \quad i=1, \ldots, 10 .
$$

Next, we will provide information about the solutions of (8). We only are interested in solutions of (8) satisfying $\left(x_{i}, y_{i}\right) \neq\left(x_{j}, y_{j}\right)$ for $i \neq j$. In what follows, these solutions will be called valid solutions.

The first two equations of system (8) can be written as

$$
\begin{aligned}
& a_{1} x_{4}^{2}+a_{1} y_{4}^{2}-a_{1} x_{4}+c_{1} y_{4}=0, \\
& a_{2} x_{4}^{2}+a_{2} y_{4}^{2}+b_{2} x_{4}+c_{2} y_{4}=0,
\end{aligned}
$$

where

$$
\begin{aligned}
a_{1}= & x_{5} y_{3}-x_{5}^{2} y_{3}+x_{3} y_{5}-x_{3}^{2} y_{5}-y_{3}^{2} y_{5}-y_{3} y_{5}^{2}, \\
c_{1}= & -x_{3} x_{5}+x_{3}^{2} x_{5}+x_{3} x_{5}^{2}-x_{3}^{2} x_{5}^{2}+x_{5} y_{3}^{2}-x_{5}^{2} y_{3}^{2}+y_{3} y_{5}+x_{3} y_{5}^{2}-x_{3}^{2} y_{5}^{2}-y_{3}^{2} y_{5}^{2}, \\
a_{2}= & x_{5} y_{3}-2 x_{3} x_{5} y_{3}+x_{5}^{2} y_{3}-x_{3} y_{5}+x_{3}^{2} y_{5}-y_{3}^{2} y_{5}+y_{3} y_{5}^{2}, \\
b_{2}= & -2 x_{3} x_{5} y_{3}+3 x_{3}^{2} x_{5} y_{3}+x_{5}^{2} y_{3}-2 x_{3} x_{5}^{2} y_{3}-x_{5} y_{3}^{3}+x_{3}^{2} y_{5}-x_{3}^{3} y_{5}-y_{3}^{2} y_{5} \\
& +3 x_{3} y_{3}^{2} y_{5}+y_{3} y_{5}^{2}-2 x_{3} y_{3} y_{5}^{2}, \\
c_{2}= & x_{3}^{2} x_{5}-x_{3}^{3} x_{5}-x_{3} x_{5}^{2}+x_{3}^{2} x_{5}^{2}-x_{5} y_{3}^{2}+3 x_{3} x_{5} y_{3}^{2}-x_{5}^{2} y_{3}^{2}+2 x_{3} y_{3} y_{5}-3 x_{3}^{2} y_{3} y_{5} \\
& +y_{3}^{3} y_{5}-x_{3} y_{5}^{2}+x_{3}^{2} y_{5}^{2}-y_{3}^{2} y_{5}^{2} .
\end{aligned}
$$

Systems of the form (9) will appear often in our analysis. We give the solution of this kind of systems in the next section, considering a generic system as (11).

Many solutions of (8) satisfy that $y_{i}=0$ for some $i=3,4$, and 5 , these solutions correspond to isochronous configurations with at least three zeros aligned. In order to simplify our computations, this case is treated separately, at Theorem 4. From now on, the solutions of (8) with $y_{i} \neq 0$ for all $i=3,4$, and 5 that are valid solutions will be called admissible solutions.

3.1. The Resolution of Systems of the Form (9). Let us consider a generic system of the form (9):

$$
\begin{aligned}
& A_{1} x^{2}+A_{1} y^{2}-A_{1} x+C_{1} y=0, \\
& A_{2} x^{2}+A_{2} y^{2}+B_{2} x+C_{2} y=0 .
\end{aligned}
$$

Note that the two equations of (11) correspond to the equations of two circles (eventually degenerated to a line) passing through the point $(0,0)$. So, $(x, y)=(0,0)$ is always a solution of (11).

After tedious but not difficult computations, we see that when $D_{1}=A_{1}\left(A_{2}+B_{2}\right)$ and $D_{2}=A_{2} C_{1}-A_{1} C_{2}$ are not simultaneously zero, then the solutions of system (11) are $(x, y)=(0,0)$ and $(x, y)=\left(x_{g}, y_{g}\right)$ with

$$
\left(x_{g}, y_{g}\right)=\left(-\frac{\left(A_{2} C_{1}-A_{1} C_{2}\right)\left(B_{2} C_{1}+A_{1} C_{2}\right)}{D_{1}^{2}+D_{2}^{2}},-\frac{A_{1}\left(A_{2}+B_{2}\right)\left(B_{2} C_{1}+A_{1} C_{2}\right)}{D_{1}^{2}+D_{2}^{2}}\right) .
$$

Solving system $D_{1}=0$ and $D_{2}=0$, we get the following conditions: $\kappa_{1}:\left\{A_{1}=0, C_{1}=0\right\}, \kappa_{2}:\left\{A_{1}=0, A_{2}=0\right\}$, and
$\kappa_{3}:\left\{B_{2}=-A_{2}, A_{2} C_{1}-A_{1} C_{2}=0\right\}$. Then, we can prove the following result. 
Lemma 1. Let $\kappa_{1}:\left\{A_{1}=0, C_{1}=0\right\}, \kappa_{2}:\left\{A_{1}=0, A_{2}=0\right\}$, and $\kappa_{3}:\left\{B_{2}=-A_{2}, A_{2} C_{1}-A_{1} C_{2}=0\right\}$. Then, the following statements hold.

(a) $(x, y)=(0,0)$ is a solution of (11) for all $A_{1}, C_{1}, A_{2}$, $B_{2}$, and $C_{2}$

(b) If neither of conditions $\kappa_{1}, \kappa_{2}$, and $\kappa_{3}$ is satisfied, then the solutions of $(11)$ are $(x, y)=(0,0)$ and $(x, y)=$ $\left(x_{g}, y_{g}\right)$ as given in (12)

(c) If condition $\kappa_{1}$ is satisfied, then the solutions of (11) are the solutions of equation $A_{2} x^{2}+A_{2} y^{2}+$ $B_{2} x+C_{2} y=0$

(d) If condition $\kappa_{1}$ is not satisfied and condition $\kappa_{2}$ is satisfied, then the solution of $(11)$ is $(x, y)=(0,0)$ when $B_{2} \neq 0$ and $(x, y)=(x, 0)$ when $B_{2}=0$

(e) If condition $\kappa_{1}$ is not satisfied and condition $\kappa_{3}$ is satisfied, then either the second equation is identically 0 or the two equations of (11) are linearly dependent

3.2. Isochronous Configurations with Three Zeros in a Line. Without loss of generality, we can assume that the zeros that are aligned are $p_{1}, p_{2}$, and $p_{3}$, so we assume that $y_{3}=0$.

We substitute $y_{3}=0$ into equations $f_{1}=0$ and $f_{2}=0$, and we get the equations:

$$
\begin{gathered}
-\left(x_{3}-1\right) x_{3} h_{1}:=-\left(x_{3}-1\right) x_{3}\left(A_{1} x_{4}^{2}+A_{1} y_{4}^{2}-A_{1} x_{4}+C_{1} y_{4}\right)=0, \\
\left(x_{3}-1\right) x_{3} h_{2}:=\left(x_{3}-1\right) x_{3}\left(A_{2} x_{4}^{2}+A_{2} y_{4}^{2}+B_{2} x_{4}+C_{2} y_{4}\right)=0,
\end{gathered}
$$

where we define $h_{1}=A_{1} x_{4}^{2}+A_{1} y_{4}^{2}-A_{1} x_{4}+C_{1} y_{4}$ and $h_{2}=$ $A_{2} x_{4}^{2}+A_{2} y_{4}^{2}+B_{2} x_{4}+C_{2} y_{4}$ with

$$
\begin{aligned}
& A_{1}=y_{5}, \\
& C_{1}=-x_{5}+x_{5}^{2}+y_{5}^{2}, \\
& A_{2}=y_{5}, \\
& B_{2}=-x_{3} y_{5}, \\
& C_{2}=-x_{3} x_{5}+x_{5}^{2}+y_{5}^{2} .
\end{aligned}
$$

The solutions with $x_{3}=1$ and $x_{3}=0$ are not valid because they correspond to $p_{3}=p_{2}$ and $p_{3}=p_{1}$, respectively. Now, we analyze the solutions of system $h_{1}=0$ and $h_{2}=0$. This is a system of the form (11); hence, from Lemma 1, if conditions $\kappa_{i}$ with $i=1,2$, and 3 are not satisfied, then the nontrivial solution of system $h_{1}=0$ and $h_{2}=0$ is

$$
\begin{aligned}
& x_{4}=x_{5}, \\
& y_{4}=-y_{5} .
\end{aligned}
$$

Condition $\kappa_{1}$ does not provide valid solutions because it is satisfied when either $\left(x_{5}, y_{5}\right)=(0,0)$ or $\left(x_{5}, y_{5}\right)=(1,0)$ (i.e., when either $p_{5}=p_{1}$ or $\left.p_{5}=p_{2}\right)$. Condition $\kappa_{2}$ is satisfied when $y_{5}=0$; under this condition, $B_{2}=0$; so, from Lemma 1 , the solution of system $h_{1}=0$ and $h_{2}=0$ is $\left(x_{4}, y_{4}\right)=(x, 0)$ with $x \in \mathbb{R}$. Finally, condition $\kappa_{3}$ is satisfied either when $x_{3}=1$, which does not provide a valid solution, or when $y_{5}=0$. This last case satisfies condition $\kappa_{2}$, so it has already been studied.

In short, system $f_{1}=0$ and $f_{2}=0$ with $y_{3}=0$ have only two valid solutions:

$$
\begin{aligned}
& s_{21}=\left\{y_{3}=0, x_{4}=x_{5}, y_{4}=-y_{5}, y_{5} \neq 0\right\}, \\
& s_{22}=\left\{y_{3}=0, y_{4}=0, y_{5}=0\right\} .
\end{aligned}
$$

By substituting $s_{21}$ into equation $f_{3}=0$, we get

$$
2 x_{3} y_{5}\left(-x_{3} x_{5}+x_{5}^{2}+x_{3} x_{5}^{2}-x_{5}^{3}-y_{5}^{2}-x_{3} y_{5}^{2}+3 x_{5} y_{5}^{2}\right)=0 \text {. }
$$

The solutions $x_{3}=0$ and $y_{5}=0$ of this equation are not valid, and the solutions of the last factor of the equation are

$$
x_{3}=\frac{x_{5}^{3}-x_{5}^{2}-3 x_{5} y_{5}^{2}+y_{5}^{2}}{x_{5}^{2}-x_{5}-y_{5}^{2}},
$$

when $x_{5}^{2}-x_{5}-y_{5}^{2} \neq 0$ and either $x_{5}=1, x_{5}=0$, or $x_{5}=$ $(1 / 2)$ when $x_{5}^{2}-x_{5}-y_{5}^{2}=0$ (or equivalently when $y_{5}=$ $\left.\pm \sqrt{x_{5}^{2}-x_{5}}\right)$. It is easy to check that the solutions with $x_{5}^{2}-$ $x_{5}-y_{5}^{2}=0$ do not provide valid solutions. On the contrary, the solution $s_{21}$ with $x_{3}$ given in (18) and the solution $s_{22}$ satisfy all equations $f_{i}=0$. Therefore, they provide isochronous configurations. In short, we have proved the following theorem.

Theorem 4. If the zeros $p_{1}, p_{2}$, and $p_{3}$ are in a line, then the configuration $\left[p_{1}, \ldots, p_{5}\right]$ is isochronous if and only if it satisfies one of the following statements:

(a) $p_{1}, p_{2}, p_{3}, p_{4}$, and $p_{5}$ are in a line

(b) $p_{1}, p_{2}$, and $p_{3}$ are in the bisector line of the segment with endpoints $p_{4}$ and $p_{5}$

In particular, if $p_{1}=0, \quad p_{2}=1, \quad p_{3}=x_{3}$, $p_{4}=x_{4}+i y_{4}$, and $p_{5}=x_{5}+i y_{5}$, then $X$ is isochronous if and only if one of the following statements holds:

(c) $y_{4}=y_{5}=0$

(d) $x_{4}=x_{5}, \quad y_{4}=-y_{5}, \quad$ and $\quad x_{3}=x_{3}^{\ell}=\left(\left(x_{5}^{3}-x_{5}^{2}-\right.\right.$ $\left.\left.3 x_{5} y_{5}^{2}+y_{5}^{2}\right) /\left(x_{5}^{2}-x_{5}-y_{5}^{2}\right)\right)$

Theorem 4 completes in some sense the results in Theorem 3.

From now on, we only will consider solutions of system (8) with $y_{3}, y_{4}$, and $y_{5} \neq 0$.

3.3. Admissible Solutions of System (8). Now we analyze the solutions of (9) that provide solutions of (8) with $p_{i} \neq p_{j}$ for $i \neq j$ and $y_{3}, y_{4}$, and $y_{5} \neq 0$, that is, those provide admissible solutions. System (9) is of the form (11) with $(x, y)=\left(x_{4}, y_{4}\right), A_{1}=a_{1}, A_{2}=a_{2}, B_{2}=b_{2}, C_{1}=c_{1}$, and $C_{2}=c_{2}$ so we will apply Lemma 1 . Here, we use the notation: 


$$
\begin{aligned}
& K_{1}:\left\{a_{1}=0, c_{1}=0\right\}, \\
& K_{2}:\left\{a_{1}=0, a_{2}=0\right\}, \\
& K_{3}:\left\{b_{2}=-a_{2}, a_{2} c_{1}-a_{1} c_{2}=0\right\} .
\end{aligned}
$$

In order to simplify our computations, we will use resultants' theory in some cases. Next, we summarize the basic properties of the resultants.

Let $P$ and $Q$ be two polynomials in the variable $x$ with leading coefficient one. Let $a_{i}, i=1,2, \ldots, n$, be the roots of $P$ and $b_{j}, j=1,2, \ldots, m$, be the roots of $Q$. The resultant of $P$ and $Q, \operatorname{Res}[P, Q]$, is the expression formed by the product of all the differences $a_{i}-b_{j}, i=1,2, \ldots, n$ and $j=1,2, \ldots, m$, see for instance $[12,13]$. The main property of the resultant is that if $P$ and $Q$ have a common solution, then necessarily $\operatorname{Res}[P, Q]=0$.

Let now $P$ and $Q$ be polynomials in the variables $(x, y)$. These polynomials can be considered as polynomials in $x$ with polynomial coefficients in $y$; then, the resultant with respect to $x, \operatorname{Res}[P, Q, x]$, is a new polynomial in the variable $y$ with the following property. If $P$ and $Q$ have a common solution $\left(x_{0}, y_{0}\right)$, then $\operatorname{Res}[P, Q, x]\left(y_{0}\right)=0$ and similarly for the variable $y$.

3.3.1. Solutions of (9) Satisfying Condition $K_{1}$. System $a_{1}=0$ and $c_{1}=0$ (condition $K_{1}$ ) can be written in the form (11) with $(x, y)=\left(x_{5}, y_{5}\right), A_{1}=C_{2}=y_{3}$, and $B_{2}=C_{1}=-A_{2}=$ $-\left(x_{3}-x_{3}^{2}-y_{3}^{2}\right)$ and can be solved by applying Lemma 1 again. Conditions $\kappa_{i}$ for $i=1,2$, and 3 provide solutions with either $p_{3}=p_{2}$ or $p_{3}=p_{1}$. The solution $\left(x_{q}, y_{q}\right)$ in Lemma 1 becomes $\left(x_{5}, y_{5}\right)=(1,0)$ (i.e., $\left.p_{2}=p_{5}\right)$. Therefore, neither of the solutions of (9) satisfying condition $K_{1}$ can provide valid solutions of (8).
3.3.2. Solutions of (9) Satisfying Condition $K_{2}$. System $a_{1}=0$ and $a_{2}=0$ (condition $K_{2}$ ) can be written again in the form (11) with $(x, y)=\left(x_{5}, y_{5}\right), A_{1}=A_{2}=y_{3}, B_{2}=y_{3}-2 x_{3} y_{3}$, $C_{1}=-\left(x_{3}-x_{3}^{2}-y_{3}^{2}\right)$, and $C_{2}=-\left(x_{3}-x_{3}^{2}+y_{3}^{2}\right)$. All conditions $\kappa_{i}$ in Lemma 1 provide solutions with $y_{3}=0$. Therefore, conditions $\kappa_{i}$ cannot provide admissible solutions of (8).

The solution $\left(x_{g}, y_{g}\right)$ in Lemma 1 becomes

$$
\left(x_{5}, y_{5}\right)=\left(x_{3}, \frac{x_{3}-x_{3}^{2}}{y_{3}}\right) \text {. }
$$

We substitute this solution into equation $f_{1}=0$, and we get an equation equivalent to

$$
\left(1-x_{3}\right) x_{3} y_{4}\left(x_{3}^{2}+y_{3}^{2}\right)\left(\left(x_{3}-1\right)^{2}+y_{3}^{2}\right)=0 .
$$

Clearly, neither of the solutions of this equation can provide admissible solutions of (8). In short, neither of the solutions of (9) satisfying condition $K_{2}$ can provide valid solutions of (8).

3.3.3. Admissible Solutions of (8) Satisfying Condition $K_{3}$. Finally, we analyze the solutions of system $F_{1}=b_{2}+a_{2}=0$ and $F_{2}=a_{2} c_{1}-a_{1} c_{2}=0$ (condition $K_{3}$ ). System $F_{1}=0$ and $F_{2}=0$ cannot be written in the form (11). We will analyze the solution of this system by using the properties of resultants.

We compute the resultant of $F_{1}$ and $F_{2}$ with respect to $x_{5}$, and we get

$$
\operatorname{Res}\left(F_{1}, F_{2}, x_{5}\right)=-2 y_{3}^{3} y_{5}^{2}\left(x_{3}^{2}+y_{3}^{2}\right)\left(\left(x_{3}^{2}-1\right)^{2}+y_{3}^{2}\right)^{4}\left(y_{3}-y_{5}\right) F_{a} \text {, }
$$

where

$$
\begin{aligned}
F_{a}= & 9 x_{3}^{6}-27 x_{3}^{5}+3 x_{3}^{4} y_{3}^{2}+18 x_{3}^{4} y_{3} y_{5}+27 x_{3}^{4}-6 x_{3}^{3} y_{3}^{2}-36 x_{3}^{3} y_{3} y_{5}-9 x_{3}^{3}-5 x_{3}^{2} y_{3}^{4} \\
& +20 x_{3}^{2} y_{3}^{3} y_{5}+18 x_{3}^{2} y_{3} y_{5}+5 x_{3} y_{3}^{4}-20 x_{3} y_{3}^{3} y_{5}+3 x_{3} y_{3}^{2}+y_{3}^{6}+2 y_{3}^{5} y_{5}-3 y_{3}^{4}+2 y_{3}^{3} y_{5} .
\end{aligned}
$$

By the properties of the resultant, we know that if $\left(x_{3}, y_{3}, x_{5}, y_{5}\right)$ is a solution of system $F_{1}=0$ and $F_{2}=0$, then the coordinates $\left(x_{3}, y_{3}, y_{5}\right)$ satisfy equation $\operatorname{Res}\left(F_{1}, F_{2}, x_{5}\right)=0$. Thus, in order to solve system $F_{1}=0$ and $F_{2}=0$, it is sufficient to find the solutions $\left(x_{3}, y_{3}, y_{5}\right)$ of $\operatorname{Res}\left(F_{1}, F_{2}, x_{5}\right)=0$ that satisfy system $F_{1}=0$ and $F_{2}=0$.

Clearly, the first four factors of $\operatorname{Res}\left(F_{1}, F_{2}, x_{5}\right)$ do not provide admissible solutions of (8). Thus, we only will consider solutions with either $y_{3}=y_{5}$ or $F_{a}=0$.
Case $y_{3}=y_{5}$ :

We substitute $y_{3}=y_{5}$ into equation $F_{1}=0$, and we get

$$
y_{5}\left(x_{5}-x_{3}\right)\left(x_{3}^{2}-2 x_{3} x_{5}-2 x_{3}+2 x_{5}-y_{5}^{2}+1\right)=0 \text {. }
$$

The first two factors of this equation do no provide admissible solutions of (8). From the last factor of the equation, we have

$$
y_{5}= \pm y_{5}^{*}= \pm \sqrt{\left(1-x_{3}\right)\left(1-x_{3}+2 x_{5}\right)} .
$$


We substitute $y_{5}= \pm y_{5}^{*}$ into equation $F_{2}=0$, and we get the following solutions:

$$
\begin{aligned}
& x_{5}=x_{3}, \\
& x_{5}=\frac{x_{3}-1}{2}, \\
& x_{3}=1, \\
& x_{5}=x_{3}-1, \\
& x_{5}=x_{5}^{*}
\end{aligned}
$$

The first solution corresponds to $p_{3}=p_{5}$, the second and the third imply $y_{5}=0$, and the fourth corresponds to $p_{3}=p_{2}$. Therefore, the unique solutions of system $F_{1}=0$ and $F_{2}=0$ that can provide admissible solutions of (8) are

$$
\begin{aligned}
& x_{5}=x_{5}^{*}=\frac{1}{2}\left(4 x_{3}-1\right), \\
& y_{5}= \pm y_{5}^{*}= \pm \sqrt{3} \sqrt{x_{3}-x_{3}^{2}} .
\end{aligned}
$$

Notice that $y_{5}^{*}$ is defined for $x_{3} \in[0,1]$ and $y_{5}^{*}=0$ when either $x_{3}=0$ or $x_{3}=1$.

We substitute $x_{5}=x_{5}^{*}$ and $y_{5}= \pm y_{5}^{*}$ into (8). Since condition $K_{3}$ is satisfied, equations $f_{1}=0$ and $f_{2}=0$ are linearly dependent, so we will work with equations $f_{1}=0$ and $f_{4}=0$ instead of equations $f_{1}=0$ and $f_{2}=0$. If $x_{5}=$ $x_{5}^{*}$ and $y_{5}= \pm y_{5}^{*}$, then system $f_{1}=0$ and $f_{4}=0$ can be written as

$$
\begin{aligned}
& \pm \frac{1}{4}\left(2 x_{3}-3\right)\left(2 x_{3}+1\right) F_{1}^{*}=0, \\
& \pm \frac{1}{8}\left(2 x_{3}-3\right)\left(2 x_{3}-1\right) F_{2}^{*}=0,
\end{aligned}
$$

where

$$
\begin{aligned}
& F_{1}^{*}=A_{1}^{*} x_{4}^{2}+A_{1}^{*} y_{4}^{2}-A_{1}^{*} x_{4}+C_{1}^{*} y_{4}=0, \\
& F_{2}^{*}=A_{2}^{*} x_{4}^{2}+A_{2}^{*} y_{4}^{2}+B_{2}^{*} x_{4}+C_{2}^{*} y_{4}=0 .
\end{aligned}
$$

Here,

$$
\begin{aligned}
& A_{1}^{*}=y_{5}^{*}, \\
& C_{1}^{*}=\mp 2\left(x_{3}-x_{3}^{2}\right), \\
& A_{2}^{*}=2 y_{5}^{*}, \\
& B_{2}^{*}=\left(1-6 x_{3}\right) y_{5}^{*}, \\
& C_{2}^{*}=\mp\left(7-10 x_{3}\right) x_{3},
\end{aligned}
$$

where the upper sign corresponds to the positive value $y_{5}^{*}$ and the lower sign corresponds to the negative value $y_{5}^{*}$. Notice that, in order to obtain the expressions $F_{1}^{*}$ and $F_{2}^{*}$, we have substituted into system $f_{1}=0$ and $f_{4}=0$ not only $x_{5}=$ $x_{5}^{*}$ and $y_{5}= \pm y_{5}^{*}$ but also $y_{5}^{2}=3\left(x_{3}-x_{2}^{2}\right), y_{5}^{3}= \pm y_{5}^{*} y_{5}^{2}$, and $y_{5}^{4}=9\left(x_{3}-x_{2}^{2}\right)^{2}$.

Next, we analyze the solutions of (28) providing admissible solutions of $(8)$. The factors $\left(2 x_{3}-3\right)$ and $\left(2 x_{3}+1\right)$ do not provide solutions in the domain of definition of $y_{5}^{*}$, and the factor $\left(2 x_{3}-1\right)$ provides solutions with $p_{5}=p_{3}$. We analyze the solutions of $F_{1}^{*}=0$ and $F_{2}^{*}=0$ by applying again Lemma 1. Conditions $\kappa_{1}$ and $\kappa_{2}$ in Lemma 1 give solutions with either $p_{3}=p_{2}$ or $p_{3}=p_{1}$, and condition $\kappa_{3}$ gives solutions with either $p_{3}=p_{2}, p_{3}=p_{1}$, or $p_{5}=p_{3}$. The solution given by $\left(x_{g}, y_{g}\right)$ in Lemma 1 becomes

$$
\left(x_{4}, y_{4}\right)=\left(x_{3}, \pm \sqrt{3} \sqrt{x_{3}-x_{3}^{2}}\right),
$$

and it corresponds to $p_{3}=p_{4}$. Therefore, the factor $y_{3}=y_{5}$ does not provide admissible solutions of (8).

Case $F_{a}=0$ :

From equation $F_{a}=0$, we get

$y_{5}=y_{5}^{\star}=-\frac{1}{2 y_{3} d}\left(y_{3}^{2}-3 x_{3}^{2}\right)\left(-3 x_{3}^{2}+6 x_{3}+y_{3}^{2}-3\right)\left(x_{3}^{2}-x_{3}+y_{3}^{2}\right)$,

where

$$
d=9 x_{3}^{4}-18 x_{3}^{3}+9 x_{3}^{2}+\left(10 x_{3}^{2}-10 x_{3}+1\right) y_{3}^{2}+y_{3}^{4} .
$$

Note that $y_{5}^{\star}$ is defined when $y_{3} \neq 0$ and $d \neq 0$.

Solutions with $d=0$ : solving equation $d=0$ with respect to $y_{3}$, we get $y_{3}= \pm \sqrt{d_{1}}$ and $y_{3}= \pm \sqrt{d_{2}}$ where

$$
\begin{aligned}
& d_{1}=\frac{1}{2}\left(-10 x_{3}^{2}+10 x_{3}-1-\sqrt{\left(2 x_{3}-1\right)^{2}\left(16 x_{3}^{2}-16 x_{3}+1\right)}\right), \\
& d_{2}=\frac{1}{2}\left(-10 x_{3}^{2}+10 x_{3}-1+\sqrt{\left(2 x_{3}-1\right)^{2}\left(16 x_{3}^{2}-16 x_{3}+1\right)}\right) .
\end{aligned}
$$

The domain of definition of $d_{1}$ and $d_{2}$ is the set

$$
D=\left(-\infty, \frac{1}{4}(2-\sqrt{3})\right] \cup\left\{\frac{1}{2}\right\} \cup\left[\frac{1}{4}(2-\sqrt{3}),+\infty\right) \text {. }
$$

On the contrary, analyzing the functions $d_{1}$ and $d_{2}$, we can see that $d_{1} \leq-(3 / 16)$ when $x_{3} \in D \backslash(1 / 2), d_{1}=(3 / 4)$ when $x_{3}=(1 / 2), d_{2}=0$ when either $x_{3}=0$ or $x_{3}=1, d_{2}<0$ when $x_{3} \in D \backslash\{(1 / 2), 0,1\}$, and $d_{2}=(3 / 4)$ when $x_{3}=(1 / 2)$. Thus, the solutions $y_{3}= \pm \sqrt{d_{1}}$ and $y_{3}= \pm \sqrt{d_{2}}$ are defined only when $x_{3}=(1 / 2)$. Therefore, the denominator of $y_{5}^{\star}$ is zero when either $y_{3}=0$ or $\left(x_{3}, y_{3}\right)=((1 / 2), \pm(\sqrt{3} / 2))$. The solution $y_{3}=0$ does not provide admissible solutions of system (8).

Now we analyze the solutions of (8) with $\left(x_{3}, y_{3}\right)=((1 / 2), \pm(\sqrt{3} / 2))$. In this case, $F_{a}$ is identically 0 . By substituting $\left(x_{3}, y_{3}\right)=((1 / 2), \pm(\sqrt{3} / 2))$ into equation $F_{1}=0$ and solving the resulting equation, we get the solutions $y_{5}= \pm y_{51}$ and $y_{5}= \pm y_{52}$ where

$$
\begin{aligned}
& y_{51}=\frac{1+\sqrt{-12 x_{5}^{2}+12 x_{5}+1}}{2 \sqrt{3}}, \\
& y_{52}=\frac{1-\sqrt{-12 x_{5}^{2}+12 x_{5}+1}}{2 \sqrt{3}} .
\end{aligned}
$$


These solutions also satisfy equation $F_{2}=0$. We substitute the solution with $\left(x_{3}, y_{3}\right)=((1 / 2), \pm(\sqrt{3} / 2))$ and $y_{5}=y_{51}$ into equations $f_{1}=0$ and $f_{4}=0$, and we obtain a system of equations of the form (9) in the variables $(x, y)=$ $\left(x_{4}, y_{4}\right)$ where the coefficients $A_{1}, C_{1}, A_{2}, B_{2}$, and $C_{2}$ depend on $x_{5}$. We solve this system by applying Lemma 1 as we have done in the previous cases, and we see that it has two unique solutions, one satisfying $p_{4}=p_{5}$ and the other one satisfying $p_{3}=p_{5}$. The same occurs with the solution $\left(x_{3}, y_{3}\right)=((1 / 2),-(\sqrt{3} / 2))$ and $y_{5}=-y_{51}$. Analyzing the cases $\left(x_{3}, y_{3}\right)=((1 / 2), \pm(\sqrt{3} / 2))$ and $y_{5}= \pm y_{52}$ in a similar way, we get three unique solutions, which satisfy $p_{4}=p_{5}, p_{1}=p_{5}$, and $p_{2}=p_{5}$, respectively. Therefore, system (8) has no valid solutions when $d=0$.

Solutions with $d \neq 0$ : assume that $\left(x_{3}, y_{3}\right) \neq((1 /$ 2), $\pm(\sqrt{3} / 2))$. We substitute $y_{5}=y_{5}^{\star}$ into equation $F_{1}=0$, and we solve the resulting equation obtaining in this way the solutions:

$$
\begin{aligned}
& y_{3}=0 \\
& x_{5}=x_{5}^{(\star, 1)}=\frac{1}{2 d}\left(12 x_{3}^{5}-21 x_{3}^{4}+8 x_{3}^{3} y_{3}^{2}+6 x_{3}^{3}-2 x_{3}^{2} y_{3}^{2}+3 x_{3}^{2}-4 x_{3} y_{3}^{4}-2 x_{3} y_{3}^{2}+3 y_{3}^{4}-y_{3}^{2}\right), \\
& x_{5}=x_{5}^{(\star, 2)}=\frac{1}{2\left(x_{3}-1\right) d}\left(15 x_{3}^{6}-57 x_{3}^{5}+13 x_{3}^{4} y_{3}^{2}+81 x_{3}^{4}-42 x_{3}^{3} y_{3}^{2}-51 x_{3}^{3}-3 x_{3}^{2} y_{3}^{4}+44 x_{3}^{2} y_{3}^{2}+12 x_{3}^{2}-x_{3} y_{3}^{4}-15 x_{3} y_{3}^{2}-y_{3}^{6}+3 y_{3}^{4}\right) .
\end{aligned}
$$

Note that the last solution is defined only when $x_{3} \neq 1$.

First, we analyze the solution $\left(x_{5}, y_{5}\right)=\left(x_{5}^{(*, 1)}, y_{5}^{\star}\right)$. It is easy to check that equation $F_{2}=0$ is always satisfied when $\left(x_{5}, y_{5}\right)=\left(x_{5}^{(\star 1)}, y_{5}^{\star}\right)$. We substitute this solution into equations $f_{1}=0$ and $f_{4}=0$, and we get

$$
\begin{aligned}
& \left(y_{3}^{2}-3 x_{3}^{2}\right)\left(-3 x_{3}^{2}+6 x_{3}+y_{3}^{2}-3\right)\left(x_{3}^{2}+y_{3}^{2}\right)\left(x_{3}^{2}-2 x_{3}+y_{3}^{2}+1\right) F_{1}^{\star}=0, \\
& \left(3 x_{3}^{2}-y_{3}^{2}\right)\left(-3 x_{3}^{2}+6 x_{3}+y_{3}^{2}-3\right)\left(x_{3}^{2}+y_{3}^{2}\right)\left(3 x_{3}^{4}-6 x_{3}^{3}+6 x_{3}^{2} y_{3}^{2}+3 x_{3}^{2}-6 x_{3} y_{3}^{2}+3 y_{3}^{4}-y_{3}^{2}\right) F_{2}^{\star}=0,
\end{aligned}
$$

where

Here,

$$
\begin{aligned}
& F_{1}^{\star}=A_{1}^{\star} x_{4}^{2}+A_{1}^{\star} y_{4}^{2}-A_{1}^{\star} x_{4}+C_{1}^{\star} y_{4}=0, \\
& F_{2}^{\star}=A_{2}^{\star} x_{4}^{2}+A_{2}^{\star} y_{4}^{2}+B_{2}^{\star} x_{4}+C_{2}^{\star} y_{4} .
\end{aligned}
$$

$$
\begin{aligned}
A_{1}^{\star}= & y_{3}, \\
C_{1}^{\star}= & -x_{3}^{2}+x_{3}-y_{3}^{2}, \\
A_{2}^{\star}= & -2 y_{3}^{2}\left(x_{3}^{2}+y_{3}^{2}-1\right) d, \\
B_{2}^{\star}= & 27 x_{3}^{9}-135 x_{3}^{8}+72 x_{3}^{7} y_{3}^{2}+270 x_{3}^{7}-288 x_{3}^{6} y_{3}^{2}-270 x_{3}^{6}+54 x_{3}^{5} y_{3}^{4}+450 x_{3}^{5} y_{3}^{2} \\
& +135 x_{3}^{5}-166 x_{3}^{4} y_{3}^{4}-342 x_{3}^{4} y_{3}^{2}-27 x_{3}^{4}+202 x_{3}^{3} y_{3}^{4}+126 x_{3}^{3} y_{3}^{2}-8 x_{3}^{2} y_{3}^{6} \\
& -114 x_{3}^{2} y_{3}^{4}-18 x_{3}^{2} y_{3}^{2}-9 x_{3} y_{3}^{8}+22 x_{3} y_{3}^{6}+23 x_{3} y_{3}^{4}+5 y_{3}^{8}-10 y_{3}^{6}+y_{3}^{4}, \\
C_{2}^{\star}= & -y_{3}\left(-27 x_{3}^{8}+108 x_{3}^{7}-80 x_{3}^{6} y_{3}^{2}-162 x_{3}^{6}+244 x_{3}^{5} y_{3}^{2}+108 x_{3}^{5}-78 x_{3}^{4} y_{3}^{4}\right. \\
& -254 x_{3}^{4} y_{3}^{2}-27 x_{3}^{4}+164 x_{3}^{3} y_{3}^{4}+88 x_{3}^{3} y_{3}^{2}-24 x_{3}^{2} y_{3}^{6}-102 x_{3}^{2} y_{3}^{4}+10 x_{3}^{2} y_{3}^{2} \\
& \left.+28 x_{3} y_{3}^{6}+12 x_{3} y_{3}^{4}-8 x_{3} y_{3}^{2}+y_{3}^{8}-10 y_{3}^{6}+5 y_{3}^{4}\right) .
\end{aligned}
$$

The first factor of equation (38) gives solutions with $p_{5}=p_{1}$, the second one gives solutions with $p_{5}=p_{2}$, and the third factor does not give real solutions. The fourth factor of the first equation of (38) gives solutions with $p_{3}=p_{2}$, and the fourth factor of the second equation gives solutions with $p_{5}=p_{3}$. Therefore, neither of them provides valid solutions. 
We solve system $F_{1}^{\star}=0$ and $F_{2}^{\star}=0$ by applying Lemma 1 again, and we get the solutions $\left(x_{4}, y_{4}\right)=(0,0)$ and $\left(x_{4}, y_{4}\right)=\left(x_{4}^{(*, 1)}, y_{4}^{(*, 1)}\right)$ with

$$
\begin{aligned}
& x_{4}^{(\star, 1)}=-\frac{1}{d}\left(x_{3}^{2}-2 x_{3}+y_{3}^{2}\right)\left(3 x_{3}^{3}-6 x_{3}^{2}+3 x_{3} y_{3}^{2}+3 x_{3}-2 y_{3}^{2}\right), \\
& y_{4}^{(\star, 1)}=\frac{1}{y_{3} d}\left(3 x_{3}^{3}-6 x_{3}^{2}+3 x_{3} y_{3}^{2}+3 x_{3}-2 y_{3}^{2}\right)\left(3 x_{3}^{3}-3 x_{3}^{2}+3 x_{3} y_{3}^{2}-y_{3}^{2}\right),
\end{aligned}
$$

when conditions $\kappa_{1}, \kappa_{2}$, and $\kappa_{3}$ are not satisfied. Conditions $\kappa_{1}$ and $\kappa_{2}$ provide solutions with $y_{3}=0$, and the condition $\kappa_{3}$ provides solutions with either $p_{5}=p_{2}, p_{5}=p_{3}, p_{3}=p_{1}$, $p_{3}=p_{2}$, or $d=0$. Therefore, these conditions cannot provide admissible solutions of (8). Finally, it is easy to check that, for all $i=1, \ldots, 10$, the equations $f_{i}=0$ are satisfied when $\left(x_{4}, y_{4}\right)=\left(x_{4}^{(*, 1)}, y_{4}^{(*, 1)}\right)$ and $\left(x_{5}, y_{5}\right)=\left(x_{5}^{(*, 1)}, y_{5}^{\star}\right)$. In short, this solution will provide admissible solutions when $\left(x_{3}, y_{3}\right)$ is such that $p_{i} \neq p_{j}$ for $i \neq j$ and $d \neq 0$.

Now, we consider the solution $\left(x_{5}, y_{5}\right)=\left(x_{5}^{(*, 2)}, y_{5}^{\star}\right)$ (assuming that $\left(x_{3}, y_{3}\right) \neq((1 / 2), \pm(\sqrt{3} / 2))$ ), and we proceed in a similar way. First, we substitute the solution $\left(x_{5}, y_{5}\right)=\left(x_{5}^{(*, 1)}, y_{5}^{\star}\right)$ into equation $F_{2}=0$, and we get the following equation:

$$
-\left(-3 x_{3}^{2}+6 x_{3}+y_{3}^{2}-3\right)^{2}\left(x_{3}^{2}+y_{3}^{2}\right)\left(x_{3}^{2}-2 x_{3}+y_{3}^{2}+1\right)\left(x_{3}^{2}-x_{3}+y_{3}^{2}\right)^{2}\left(3 x_{3}^{2}-3 x_{3}+y_{3}^{2}\right) G^{\star}=0,
$$

where

$$
G^{\star}=-3 x_{3}^{6}+24 x_{3}^{5}-5 x_{3}^{4} y_{3}^{2}-54 x_{3}^{4}+32 x_{3}^{3} y_{3}^{2}+48 x_{3}^{3}-x_{3}^{2} y_{3}^{4}-44 x_{3}^{2} y_{3}^{2}-15 x_{3}^{2}+8 x_{3} y_{3}^{4}+16 x_{3} y_{3}^{2}+y_{3}^{6}-6 y_{3}^{4}+y_{3}^{2} \text {. }
$$

Clearly, the second factor of equation $F_{2}=0$ does not provide real solutions, the first and fourth factors provide solutions with $p_{5}=p_{1}$, the third provides solutions with $p_{3}=p_{2}$, and the fifth provides solutions with $p_{5}=p_{3}$. Therefore, the unique factor that can provide admissible solutions of (8) is $G^{\star}$.

It is not difficult to check that

$$
x_{5}^{(\star, 2)}=x_{5}^{(\star, 1)}-\frac{G^{\star}}{2\left(x_{3}-1\right) d} .
$$

So, if $x_{3} \neq 1$ and $G^{\star}=0$, then $x_{5}^{(\star, 2)}=x_{5}^{(\star, 1)}$, and this is the case we have just been studied. Furthermore, if $x_{3}=1$, then equation $F_{1}=0$ has only the two solutions $y_{3}=0$ and $x_{5}=x_{5}^{(*, 1)}$. Since the solution $x_{5}^{(*, 2)}=x_{5}^{(*, 1)}$ is defined when $x_{3}=1$, we do not need to consider this case separately.

3.3.4. Admissible Solutions of (8) that Do Not Satisfy Any Condition $K_{i}$. From Lemma 1, when neither of conditions
$K_{i}$ is satisfied, the solutions of system $f_{1}=0$ and $f_{2}=0$ are $\left(x_{4}, y_{4}\right)=(0,0)$ and $\left(x_{4}, y_{4}\right)=\left(x_{40}, y_{40}\right)$ with

$$
\left(x_{40}, y_{40}\right)=\left(x_{g}, y_{g}\right) \text {, }
$$

where $\left(x_{g}, y_{g}\right)$ is defined in Lemma 1 and $A_{1}=a_{1}, A_{2}=a_{2}$, $B_{2}=b_{2}, C_{1}=c_{1}$, and $C_{2}=c_{2}$ are defined in (9). We substitute the solution $\left(x_{4}, y_{4}\right)=\left(x_{40}, y_{40}\right)$ into equations $f_{i}=$ 0 for $i=3, \ldots, 10$, we factorize the resulting equations, and we drop the denominators obtaining a new system of polynomial equations:

$$
g_{i}=0, \quad i=3, \ldots, 10,
$$

where the function $g_{5}$ is identically zero. Next, we analyze the solutions of (46).

The factorization of $g_{7}$ consists of two factors:

$$
g_{7}=2 \widetilde{g}_{71} \widetilde{g}_{72},
$$

where 


$$
\begin{aligned}
& \tilde{g}_{71}=x_{3}^{3} y_{5}-3 x_{3}^{2} x_{5} y_{3}-2 x_{3}^{2} y_{5}+2 x_{3} x_{5}^{2} y_{3}+4 x_{3} x_{5} y_{3}-3 x_{3} y_{3}^{2} y_{5}+2 x_{3} y_{3} y_{5}^{2} \\
& +x_{3} y_{5}-2 x_{5}^{2} y_{3}+x_{5} y_{3}^{3}-x_{5} y_{3}+2 y_{3}^{2} y_{5}-2 y_{3} y_{5}^{2} \\
& \tilde{g}_{72}=\left(2 x_{3}-1\right) y_{3}^{3} y_{5}^{7}+y_{3}^{2}\left(3 x_{3}^{3}+3 x_{5} x_{3}^{2}-6 x_{3}^{2}-y_{3}^{2} x_{3}-3 x_{5} x_{3}+3 x_{3}-3 x_{5} y_{3}^{2}+2 y_{3}^{2}\right) y_{5}^{6} \\
& =+y_{3}\left(6 x_{5} x_{3}^{4}-3 x_{3}^{4}-4 y_{3}^{2} x_{3}^{3}-12 x_{5} x_{3}^{3}+6 x_{3}^{3}-6 x_{5} y_{3}^{2} x_{3}^{2}+9 y_{3}^{2} x_{3}^{2}+6 x_{5} x_{3}^{2}-3 x_{3}^{2}\right. \\
& \left.-4 y_{3}^{4} x_{3}-2 x_{5}^{2} y_{3}^{2} x_{3}+8 x_{5} y_{3}^{2} x_{3}-6 y_{3}^{2} x_{3}+4 x_{5} y_{3}^{4}+x_{5}^{2} y_{3}^{2}\right) y_{5}^{5}+\left(-x_{3}^{7}+3 x_{5} x_{3}^{6}+2 x_{3}^{6}\right. \\
& +y_{3}^{2} x_{3}^{5}-9 x_{5} x_{3}^{5}-9 x_{5} y_{3}^{2} x_{3}^{4}+2 y_{3}^{2} x_{3}^{4}+9 x_{5} x_{3}^{4}-2 x_{3}^{4}+5 y_{3}^{4} x_{3}^{3}-9 x_{5}^{2} y_{3}^{2} x_{3}^{3}+27 x_{5} y_{3}^{2} x_{3}^{3} \\
& -9 y_{3}^{2} x_{3}^{3}-3 x_{5} x_{3}^{3}+x_{3}^{3}-11 x_{5} y_{3}^{4} x_{3}^{2}-2 y_{3}^{4} x_{3}^{2}+5 x_{5}^{3} y_{3}^{2} x_{3}^{2}+6 x_{5}^{2} y_{3}^{2} x_{3}^{2}-18 x_{5} y_{3}^{2} x_{3}^{2} \\
& +6 y_{3}^{2} x_{3}^{2}+3 y_{3}^{6} x_{3}+11 x_{5}^{2} y_{3}^{4} x_{3}+3 y_{3}^{4} x_{3}-5 x_{5}^{3} y_{3}^{2} x_{3}+3 x_{5}^{2} y_{3}^{2} x_{3}+x_{5} y_{3}^{6}-2 y_{3}^{6}-5 x_{5}^{3} y_{3}^{4} \\
& \left.+2 x_{5}^{2} y_{3}^{4}-3 x_{5} y_{3}^{4}\right) y_{5}^{4}+y_{3}\left(6 x_{5} x_{3}^{6}-3 x_{3}^{6}-18 x_{5}^{2} x_{3}^{5}+4 x_{3}^{5}+8 x_{5}^{3} x_{3}^{4}+33 x_{5}^{2} x_{3}^{4}\right. \\
& +10 x_{5} y_{3}^{2} x_{3}^{4}-5 y_{3}^{2} x_{3}^{4}-18 x_{5} x_{3}^{4}+x_{3}^{4}-16 x_{5}^{3} x_{3}^{3}-12 x_{5}^{2} x_{3}^{3}-12 x_{5}^{2} y_{3}^{2} x_{3}^{3}-8 x_{5} y_{3}^{2} x_{3}^{3} \\
& +4 y_{3}^{2} x_{3}^{3}+12 x_{5} x_{3}^{3}-2 x_{3}^{3}+2 x_{5} y_{3}^{4} x_{3}^{2}-y_{3}^{4} x_{3}^{2}+8 x_{5}^{3} x_{3}^{2}-3 x_{5}^{2} x_{3}^{2}+12 x_{5}^{3} y_{3}^{2} x_{3}^{2} \\
& +12 x_{5} y_{3}^{2} x_{3}^{2}-3 y_{3}^{2} x_{3}^{2}+6 x_{5}^{2} y_{3}^{4} x_{3}-8 x_{5} y_{3}^{4} x_{3}-10 x_{5}^{4} y_{3}^{2} x_{3}+8 x_{5}^{3} y_{3}^{2} x_{3}-12 x_{5}^{2} y_{3}^{2} x_{3} \\
& \left.-2 x_{5} y_{3}^{6}+y_{3}^{6}+4 x_{5}^{3} y_{3}^{4}-9 x_{5}^{2} y_{3}^{4}+6 x_{5} y_{3}^{4}+5 x_{5}^{4} y_{3}^{2}-4 x_{5}^{3} y_{3}^{2}+3 x_{5}^{2} y_{3}^{2}\right) y_{5}^{3} \\
& +\left(x_{5}-1\right) x_{5}\left(x_{3}^{7}-x_{5} x_{3}^{6}-3 x_{3}^{6}-y_{3}^{2} x_{3}^{5}+3 x_{5} x_{3}^{5}+3 x_{3}^{5}+11 x_{5} y_{3}^{2} x_{3}^{4}-3 y_{3}^{2} x_{3}^{4}-3 x_{5} x_{3}^{4}\right. \\
& -x_{3}^{4}-5 y_{3}^{4} x_{3}^{3}-11 x_{5}^{2} y_{3}^{2} x_{3}^{3}-11 x_{5} y_{3}^{2} x_{3}^{3}+7 y_{3}^{2} x_{3}^{3}+x_{5} x_{3}^{3}+9 x_{5} y_{3}^{4} x_{3}^{2}+3 y_{3}^{4} x_{3}^{2}+x_{5}^{3} y_{3}^{2} x_{3}^{2} \\
& +15 x_{5}^{2} y_{3}^{2} x_{3}^{2}-3 x_{5} y_{3}^{2} x_{3}^{2}-3 y_{3}^{2} x_{3}^{2}-3 y_{3}^{6} x_{3}+9 x_{5}^{2} y_{3}^{4} x_{3}-18 x_{5} y_{3}^{4} x_{3}-x_{5}^{3} y_{3}^{2} x_{3}-4 x_{5}^{2} y_{3}^{2} x_{3} \\
& \left.+3 x_{5} y_{3}^{2} x_{3}-3 x_{5} y_{3}^{6}+3 y_{3}^{6}-x_{5}^{3} y_{3}^{4}-3 x_{5}^{2} y_{3}^{4}+6 x_{5} y_{3}^{4}\right) y_{5}^{2}-\left(x_{5}-1\right)^{2} x_{5}^{2} y_{3}\left(2 x_{3}^{5}-2 x_{5} x_{3}^{4}\right. \\
& -4 x_{3}^{4}+8 y_{3}^{2} x_{3}^{3}+4 x_{5} x_{3}^{3}+2 x_{3}^{3}-18 x_{5} y_{3}^{2} x_{3}^{2}-3 y_{3}^{2} x_{3}^{2}-2 x_{5} x_{3}^{2}+6 y_{3}^{4} x_{3}+6 x_{5}^{2} y_{3}^{2} x_{3} \\
& \left.+12 x_{5} y_{3}^{2} x_{3}-3 y_{3}^{4}-3 x_{5}^{2} y_{3}^{2}-2 x_{5} y_{3}^{2}\right) y_{5}+\left(x_{5}-1\right)^{3} x_{5}^{3} y_{3}^{2}\left(x_{3}^{3}-x_{5} x_{3}^{2}-x_{3}^{2}-3 y_{3}^{2} x_{3}\right. \\
& \left.+x_{5} x_{3}+x_{5} y_{3}^{2}+y_{3}^{2}\right) \text {. }
\end{aligned}
$$

Then, the solutions of (46) must satisfy either $\widetilde{g}_{71}=0$ or $\tilde{g}_{72}=0$. We see that factor $\widetilde{g}_{72}$ is common to all the functions $g_{i}$ for $i=3,4,6,7,8,9$, and 10 . Therefore, all the solutions $\left(x_{3}, y_{3}, x_{5}, y_{5}\right)$ of equation $\widetilde{g}_{72}=0$ that do not satisfy conditions $K_{i}$ with $i=1,2$, and 3 provide isochronous configurations with $\left(x_{4}, y_{4}\right)=\left(x_{40}, y_{40}\right)$.

It is easy to check that $\widetilde{g}_{71}=-F_{1}$. Therefore, all the solutions of equation $\widetilde{g}_{71}=0$ satisfy condition $K_{3}$, and the solution $\left(x_{4}, y_{4}\right)=\left(x_{40}, y_{40}\right)$ is not defined in this case.

Remark 1. Equation $\widetilde{g}_{72}=0$ has at most seven different solutions $y_{5}=y_{5}\left(x_{3}, y_{3}, x_{5}\right)$ with $y_{3} \neq 0$ when $x_{3} \neq(1 / 2)$, at most six different solutions $y_{5}=y_{5}\left(y_{3}, x_{5}\right)$ with $y_{3} \neq 0$ when $x_{3}=(1 / 2)$ and $x_{5} \neq(1 / 2)$, and solution $\left(x_{3}, y_{3}\right)=((1 /$ $\left.2), y_{3}\right)$ and $\left(x_{5}, y_{5}\right)=\left((1 / 2), y_{5}\right)$ when $x_{3}=x_{5}=(1 / 2)$.

Indeed, if $\left(2 x_{3}-1\right) y_{3}^{3} \neq 0$, then $\tilde{g}_{72}$ is a polynomial of degree 7 in the variable $y_{5}$; therefore, there could exist up to seven different real solutions $y_{5}=y_{5}\left(x_{3}, y_{3}, x_{5}\right)$ of $\tilde{g}_{72}=0$. The coefficient of degree 7 of $\widetilde{g}_{72},\left(2 x_{3}-1\right) y_{3}^{3}$, is equal to zero when either $x_{3}=(1 / 2)$ or $y_{3}=0$. Here, we are not interested in solutions with $y_{3}=0$. So, $\tilde{g}_{72}$ becomes a polynomial of degree 6 with at most 6 solutions when $x_{3}=$ $(1 / 2)$. If $x_{3}=(1 / 2)$, then the coefficient of degree 6 of $\tilde{g}_{72}$ is

$$
-\frac{3}{8}\left(2 x_{5}-1\right) y_{3}^{2}\left(4 y_{3}^{2}+1\right)
$$

which becomes zero when $x_{5}=(1 / 2)$. But if $x_{3}=(1 / 2)$ and $x_{5}=(1 / 2)$, then $\widetilde{g}_{72}=0$.

We note that the solution with $\left(x_{3}, y_{3}\right)$, $\left(x_{4}, y_{4}\right)=\left(x_{4}^{(*, 1)}, y_{4}^{(\star, 1)}\right)$, and $\left(x_{5}, y_{5}\right)=\left(x_{5}^{(*, 1)}, y_{5}^{\star}\right)$ always satisfies equation $\widetilde{g}_{72}=0$, but in this case, the solution $\left(x_{4}, y_{4}\right)=\left(x_{40}, y_{40}\right)$ is not defined because $D_{1}^{2}+D_{2}^{2}=0$ (or equivalently, because conditions $K_{i}=0$ are satisfied for some $i=1,2$, and 3). In short, we have proved the following theorem.

Theorem 5. Let $p_{1}=0, p_{2}=1, p_{3}=x_{3}+i y_{3}, p_{4}=x_{4}+i y_{4}$, and $p_{5}=x_{5}+i y_{5}$. Then, we can have the following families of solutions of (8) with $p_{i} \neq p_{j}$ for $i \neq j$ providing isochronous configurations with $y_{i} \neq 0$ for $i=3,4$, and 5 .

(a) Up to seven different three-parameter families of solutions with $\left(x_{4}, y_{4}\right)=\left(x_{40}, y_{40}\right)$ (see (45)) and $y_{5}=y_{5}\left(x_{3}, y_{3}, x_{5}\right)$ satisfying equation $\tilde{g}_{72}=0$ when $x_{3} \neq(1 / 2)$

(b) Up to six different two-parameter families of solutions with $\left(x_{4}, y_{4}\right)=\left(x_{40}, y_{40}\right)$ and $y_{5}=y_{5}\left(y_{3}, x_{5}\right)$ satisfying equation $\widetilde{g}_{72}=0$ when $x_{3}=(1 / 2)$ and $x_{5} \neq(1 / 2)$ 
(c) The two-parameter family of solutions with $\left(x_{4}, y_{4}\right)=$ $\left(x_{40}, y_{40}\right)=\left((1 / 2),\left(\left(y_{3}+y_{5}\right) /\left(4 y_{3} y_{5}-1\right)\right)\right),\left(x_{3}\right.$, $\left.y_{3}\right)=\left((1 / 2), y_{3}\right)$, and $\left(x_{5}, y_{5}\right)=\left((1 / 2), y_{5}\right)$

(d) The two-parameter family with $\left(x_{4}, y_{4}\right)=\left(x_{4}^{(\star, 1)}\right.$, $\left.y_{4}^{(*, 1)}\right)$ and $\left(x_{5}, y_{5}\right)=\left(x_{5}^{(*, 1)}, y_{5}^{\star}\right)$, see (32), (37), and (41)

Notice that the solution $\left(x_{4}, y_{4}\right)=\left(x_{40}, y_{40}\right)$ is defined only for solutions of $\tilde{g}_{72}=0$ that do not satisfy any of conditions $K_{1}, K_{2}$, and $K_{3}$ defined in (19). The solution in (d) is defined when $\left(x_{3}, y_{3}\right) \neq((1 / 2), \pm(\sqrt{3} / 2))$.

Theorem 4 gives a complete description of the isochronous configurations with $y_{i}=0$ for some $i=3,4$, and 5 , and Theorem 5 gives a complete description of the isochronous configurations with $y_{i} \neq 0$ for all $i=3,4$, and 5 . Hence, we have a complete description of all the isochronous configurations of the vector fields of degree 5, which is summarized in Theorem 6.

We can see that all the zeros given in Theorem 4 satisfy equation $\tilde{g}_{72}=0$. The zeros given by Theorem 4 (c) do not provide solutions with $\left(x_{4}, y_{4}\right)=\left(x_{40}, y_{40}\right)$ because in this case, $D_{1}^{2}+D_{2}^{2}=0$. This does not happen with the zeros given by Theorem 4 (d). Moreover, we can see that if $y_{3}=0$ and $x_{3}=x_{3}^{\ell}$, then $\left(x_{40}, y_{40}\right)=\left(x_{5},-y_{5}\right)$. Hence, statement $(\mathrm{d})$ of Theorem 4 can be included in either statement (a), (b), or (c) of Theorem 5 if we do not consider the assumption $y_{i} \neq 0$ for all $i=3,4$, and 5 .

Theorem 6. Let $p_{1}=0, p_{2}=1, p_{3}=x_{3}+i y_{3}, p_{4}=x_{4}+i y_{4}$, and $p_{5}=x_{5}+i y_{5}$. The only solutions of (8) with $p_{i} \neq p_{j}$ for $i \neq j$ providing isochronous configurations are the following.

(a) $y_{3}=y_{4}=y_{5}=0$

(b) $\left(x_{4}, y_{4}\right)=\left(x_{40}, y_{40}\right)$ (see (45)) and $y_{5}=y_{5}\left(x_{3}, y_{3}\right.$, $x_{5}$ ) is a solution of equation $\tilde{g}_{72}=0$ that does not satisfy any of conditions $K_{1}, K_{2}$, and $K_{3}$ defined in (19)

(c) $\left(x_{4}, y_{4}\right)=\left(x_{4}^{(\star, 1)}, y_{4}^{(\star, 1)}\right)$ and $\left(x_{5}, y_{5}\right)=\left(x_{5}^{(\star, 1)}, y_{5}^{\star}\right)$, see (32), (37), and (41)

Remark 2. Using the symmetries of the configuration, it is not difficult to see that if $p_{1}=0, p_{2}=1, p_{3}=x_{3}+y_{3} i$, $p_{4}=x_{4}+y_{4} i$, and $p_{5}=x_{5}+y_{5} i$ are an isochronous configuration, then so is the configuration $p_{1}=0, p_{2}=1$, $p_{3}=x_{3}-y_{3} i, p_{4}=x_{4}-y_{4} i$, and $p_{5}=x_{5}-y_{5} i$, the configuration $p_{1}=0, \quad p_{2}=1, \quad p_{3}=1-x_{3}+y_{3} i, \quad p_{4}=1-x_{4}+$ $y_{4} i$, and $p_{5}=1-x_{5}+y_{5} i$, and the configuration $p_{3}=$ $1-x_{3}-y_{3} i, p_{4}=1-x_{4}-y_{4} i$, and $p_{5}=1-x_{5}-y_{5} i$. So, it is sufficient to analyze the configurations with $x_{3} \leq(1 / 2)$ any $y_{3} \geq 0$.

\section{Isochronous Configurations with $n=5$ in Some Particular Cases}

In the previous section, more precisely in Theorem 6, we have given a complete description of the isochronous configurations with $n=5$. Here, we will analyze the isochronous configurations for some particular configurations of zeros.

4.1. Four Zeros at the Vertices of a Parallelogram. Without loss of generality, we can assume that the segment joining $p_{1}$ and $p_{2}$ is an edge of the parallelogram; then, the remaining vertices of the parallelogram can be taken as $p_{3}=$ $x_{3}+y_{3} i$ and $p_{5}=x_{3}+1+y_{3} i$ with $y_{3}>0$. The assumption $y_{3}>0$ is not restrictive by Remark 2 .

Since $y_{3} \neq 0$, all isochronous configurations of this type are given by Theorem 5 . First, we see that the solution with $\left(x_{4}, y_{4}\right)=\left(x_{4}^{(\star, 1)}, y_{4}^{(\star, 1)}\right)$ and $\left(x_{5}, y_{5}\right)=\left(x_{5}^{(*, 1)}, y_{5}^{\star}\right)$ does not provide isochronous configurations with four zeros at the vertices of a parallelogram. Indeed, if the configuration of $p_{1}, p_{2}, p_{3}$, and $p_{5}$ is a parallelogram, then $\left(x_{3}, y_{3}\right)$ satisfies equations $x_{5}^{(\star, 1)}=x_{3}+1$ and $y_{5}^{\star}=y_{3}$, and this system of equations does not have real solutions.

Now we substitute $\left(x_{3}, y_{3}, x_{5}, y_{5}\right)=\left(x_{3}, y_{3}, x_{3}+1, y_{3}\right)$ into equation $\tilde{g}_{72}=0$, and we get

$$
-4 y_{3}^{2}\left(x_{3}^{2}+y_{3}^{2}-1\right)\left(x_{3}^{2}+y_{3}^{2}\right)^{2}=0 \text {. }
$$

This equation has a unique real solution with $y_{3}>0$, $y_{3}=\sqrt{1-x_{3}^{2}}$. By substituting this solution into the expression of $\left(x_{40}, y_{40}\right)$, we get

$$
\left(x_{4}, y_{4}\right)=\left(x_{40}, y_{40}\right)=\left(\frac{x_{3}+1}{2}, \frac{\sqrt{1-x_{3}^{2}}}{2}\right) \text {. }
$$

In short, we have a unique one parameter family of configurations with four zeros at the vertices of a parallelogram and $y_{3}>0$. It is given by the solution

$$
\begin{aligned}
& \left(x_{3}, y_{3}\right)=\left(x_{3}, \sqrt{1-x_{3}^{2}}\right), \\
& \left(x_{4}, y_{4}\right)=\left(\frac{x_{3}+1}{2}, \frac{\sqrt{1-x_{3}^{2}}}{2}\right), \\
& \left(x_{5}, y_{5}\right)=\left(x_{3}+1, \sqrt{1-x_{3}^{2}}\right) .
\end{aligned}
$$

It is easy to check that, in this family of configurations, $p_{1}, p_{2}, p_{3}$, and $p_{5}$ are at the vertices of a rhombus (degenerated to a square when $x_{3}=0$ ) and $p_{4}$ is at its center. These configurations correspond to the ones given by Theorem 3(e), so its phase portrait has the star topology.

In short, we have proved the following result, which in some sense can be thought as a generalization of the statement (b) of Theorem 3.

Lemma 2. Assume that the configuration $\left[p_{1}, \ldots, p_{5}\right]$ is isochronous. The following statements hold.

(a) If the zeros $p_{1}, p_{2}, p_{3}$, and $p_{5}$ are at the vertices of a parallelogram, then this parallelogram is a rhombus and $p_{4}$ is at the center of the rhombus.

(b) There exist a unique configuration with four zeros at the vertices of a given rhombus. Assuming that the 
vertices of the rhombus are $p_{1}=0, p_{2}=1, p_{3}=$ $x_{3}+i \sqrt{1-x_{3}^{2}}$, and $p_{5}=x_{3}+1+i \sqrt{1-x_{3}^{2}}$, the configuration satisfies

$$
p_{4}=\frac{x_{3}+1}{2}+i \frac{\sqrt{1-x_{3}^{2}}}{2} \text {. }
$$

Notice that the rhombus is a square when $x_{3}=0$.

(c) The phase portrait associated to the configuration $\left[p_{1}, \ldots, p_{5}\right]$ has the star topology.

4.2. Four Zeros at the Vertices of an Isosceles Trapezoid. Without loss of generality, we can assume that the segment joining $p_{1}$ and $p_{2}$ is an edge of the isosceles trapezoid; then, the remaining vertices of the trapezoid can be taken as $p_{3}=$ $x_{3}+y_{3} i$ and $p_{5}=1-x_{3}+y_{3} i$. As in the previous case, here we have assumed that $y_{3}>0$, and this is not restrictive by Remark 2.

As in the previous section, since $y_{3} \neq 0$, all isochronous configurations of this type are given by Theorem 5 . First, we analyze the solution with $\left(x_{4}, y_{4}\right)=\left(x_{4}^{(\star, 1)}, y_{4}^{(\star, 1)}\right)$ and $\left(x_{5}, y_{5}\right)=\left(x_{5}^{(\star, 1)}, y_{5}^{\star}\right)$. By imposing that the configuration is an isosceles trapezoid, we get the following equations:

$$
\begin{gathered}
x_{5}^{(\star, 1)}=1-x_{3}, \\
y_{5}^{\star}=y_{3} .
\end{gathered}
$$

Solving this system of equations, we get the solutions

$$
\left(x_{3}, y_{3}\right)=\left(\frac{1}{2}, \pm \frac{1}{2 \sqrt{3}}\right)
$$

which is not a valid solution because $p_{3}=p_{5}$.

Now we substitute $\left(x_{3}, y_{3}, x_{5}, y_{5}\right)=\left(x_{3}, y_{3}, 1-x_{3}, y_{3}\right)$ into equation $\tilde{g}_{72}=0$, and we get

$-4\left(2 x_{3}-1\right)^{3} y_{3}^{4}\left(3 x_{3}^{4}-6 x_{3}^{3}+2 x_{3}^{2} y_{3}^{2}+3 x_{3}^{2}-2 x_{3} y_{3}^{2}-y_{3}^{4}+y_{3}^{2}\right)=0$.

The solution with $x_{3}=(1 / 2)$ is not possible because it corresponds to $p_{3}=p_{5}$. The last factor in (56) provides a unique real solution with $y_{3}>0$, and the solution

$$
y_{3}=y_{3}^{t}=\sqrt{x_{3}^{2}-x_{3}+\frac{1}{2} \beta+\frac{1}{2}}
$$

where

$$
\beta=\sqrt{16 x_{3}^{4}-32 x_{3}^{3}+20 x_{3}^{2}-4 x_{3}+1} \text {. }
$$

Substituting this solution into $\left(x_{40}, y_{40}\right)$, we get

$$
\left(x_{40}, y_{40}\right)=\left(x_{40}^{t}, y_{40}^{t}\right)=\left(\frac{1}{2}, \frac{\left(1+4\left(x_{3}-1\right) x_{3}-\beta\right) \sqrt{1+2\left(x_{3}-1\right) x_{3}+\beta}}{4 \sqrt{2} x_{3}\left(x_{3}-1\right)}\right) \text {. }
$$

So, we have a unique one parameter family of isosceles trapezoid configurations which is given by

$$
\begin{aligned}
& \left(x_{3}, y_{3}\right)=\left(x_{3}, y_{3}^{t}\right), \\
& \left(x_{4}, y_{4}\right)=\left(x_{40}^{t}, y_{40}^{t}\right), \\
& \left(x_{5}, y_{5}\right)=\left(1-x_{3}, y_{3}^{t}\right),
\end{aligned}
$$

which are defined for $x_{3} \neq(1 / 2)$. It is not difficult to check that $y_{3}^{t} \geq y_{40}^{t}>0$ for all $x_{3} \in \mathbb{R} \backslash\{0,1\}$ and that $y_{3}^{t}=y_{40}^{t}$ when $x_{3}=(1 / 2)$. In short, we have proved the following result.

Lemma 3. Assume that the configuration $\left[p_{1}, \ldots, p_{5}\right]$ is isochronous. The following statements hold.

(a) If the zeros $p_{1}, p_{2}, p_{3}$, and $p_{5}$ are at the vertices of an isosceles trapezoid, then $p_{4}$ is at the interior of the trapezoid on its axis of symmetry.

(b) There exist a unique configuration with four zeros at the vertices of a given isosceles trapezoid. Assuming that the vertices of the trapezoid are $p_{1}=0, p_{2}=1$, $p_{3}=x_{3}+i y_{3}$, and $p_{5}=1-x_{3}+i y_{3}$, the configuration satisfies $y_{3}=y_{3}^{t}$ with $y_{3}^{t}$ given in (57) and $p_{4}=$ $x_{40}^{t}+i y_{40}^{t}$ with $\left(x_{40}^{t}, y_{40}^{t}\right)$ given in (59).
We have plotted the phase portrait of the isochronous vector fields associated to the configurations given by Lemma 3(b) for many values of $x_{3}$. After analyzing the obtained results, we conjecture that all the isochronous configurations given by Lemma 3 (b) have a phase portrait with the star topology.

4.3. Two Zeros on the Line Orthogonal to the Line Passing through Other Two Zeros. In Theorem 4, we have proved that if $p_{1}, p_{2}$, and $p_{3}$ are in a line $L$, then $p_{4}$ and $p_{5}$ are either in the same line $L$ or $L$ is the bisector line of the segment with endpoints $p_{4}$ and $p_{5}$. Now we prove the following more generic result.

Lemma 4. Assume that the configuration $\left[p_{1}, \ldots, p_{5}\right]$ is isochronous. If $p_{1}$ and $p_{2}$ are in a line $L$ and $p_{3}$ and $p_{5}$ are in a line orthogonal to $L$ and $L^{\prime}$, then either $p_{4}$ is in $L$ and $L$ is the bisector line of the segment with endpoints $p_{3}$ and $p_{5}$ or $p_{4}$ is in $L^{\prime}$ and $L^{\prime}$ is the bisector line of the segment with endpoints $p_{1}$ and $p_{2}$.

Proof. Assume that $p_{1}=0, p_{2}=1, p_{3}=x_{3}+y_{3} i$, and $p_{5}=$ $x_{3}+y_{5} i$ with $y_{3}>0$. This is not restrictive by Remark 2 . Let $L$ 
be the line passing through $p_{1}$ and $p_{2}$ and $L^{\prime}$ be the line passing through $p_{3}$ and $p_{5}$.

Since $y_{3} \neq 0$, all the isochronous configurations of this type are given by Theorem 5 . The equation $\widetilde{g}_{72}=0$ evaluated at $x_{5}=x_{3}$ becomes

$$
\left(1-2 x_{3}\right)\left(y_{3}-y_{5}\right)^{3}\left(y_{3}+y_{5}\right)\left(x_{3}^{2}-x_{3}+y_{3} y_{5}\right)^{3}=0 .
$$

We note that the solution of (61) with $y_{3}=y_{5}$ does not provide valid solutions of (8) because it corresponds to $p_{3}=p_{5}$. If $y_{5}=\left(x_{3}-x_{3}^{2}\right) / y_{3}$, then condition $K_{2}$ is satisfied, and therefore, the solution of (8) with $\left(x_{4}, y_{4}\right)=\left(x_{40}, y_{40}\right)$ is not defined. The solutions of (61) $x_{3}=(1 / 2)$ and $y_{5}=-y_{3}$ provide valid solutions of (8) with $\left(x_{4}, y_{4}\right)=\left(x_{40}, y_{40}\right)$ which are given by

$$
\begin{aligned}
& \left(x_{3}, y_{3}\right)=\left(\frac{1}{2}, y_{3}\right), \\
& \left(x_{4}, y_{4}\right)=\left(\frac{1}{2}, \frac{y_{3}+y_{5}}{4 y_{3} y_{5}-1}\right), \\
& \left(x_{5}, y_{5}\right)=\left(\frac{1}{2}, y_{5}\right), \\
& \left(x_{3}, y_{3}\right)=\left(x_{3}, y_{3}\right), \\
& \left(x_{4}, y_{4}\right)=\left(\frac{x_{3}^{3}-x_{3}^{2}-3 x_{3} y_{3}^{2}+y_{3}^{2}}{x_{3}^{2}-x_{3}-y_{3}^{2}}, 0\right), \\
& \left(x_{5}, y_{5}\right)=\left(x_{3},-y_{3}\right),
\end{aligned}
$$

respectively. In the first solution, $p_{4}$ is on $L^{\prime}$ and $L^{\prime}$ is the bisector line of the segment with endpoints $p_{1}$ and $p_{2}$. In the second solution, $p_{4}$ is on $L$ and $L$ is the bisector line of the segment with endpoints $p_{3}$ and $p_{5}$. Notice that the solutions given in (62) correspond to the configuration given in Theorem 3(d).

Finally, we analyze the solutions of (8) with $\left(x_{4}, y_{4}\right)=$ $\left(x_{4}^{(*, 1)}, y_{4}^{(*, 1)}\right)$ and $\left(x_{5}, y_{5}\right)=\left(x_{5}^{(*, 1)}, y_{5}^{\star}\right)$. When $x_{5}=x_{3}$, equation $x_{5}^{(*, 1)}=x_{3}$ is equivalent to

$$
\left(1-2 x_{3}\right)\left(3 x_{3}^{4}-6 x_{3}^{3}+6 x_{3}^{2} y_{3}^{2}+3 x_{3}^{2}-6 x_{3} y_{3}^{2}+3 y_{3}^{4}-y_{3}^{2}\right)=0 .
$$

We can see that all the solutions of the second factor provide solutions of (8) with $y_{5}=y_{3}$ which are not valid. The solution $x_{3}=(1 / 2)$ provides the following solution:

$$
\begin{aligned}
& \left(x_{3}, y_{3}\right)=\left(\frac{1}{2}, y_{3}\right), \\
& \left(x_{4}, y_{4}\right)=\left(\frac{1}{2},-\frac{1}{4 y_{3}}\right), \\
& \left(x_{5}, y_{5}\right)=\left(\frac{1}{2}, \frac{1-4 y_{3}^{2}}{8 y_{3}}\right) .
\end{aligned}
$$

In this solution, $p_{4}$ is on $L^{\prime}$ and $L^{\prime}$ is the bisector line of the segment with endpoints $p_{1}$ and $p_{2}$. This completes the proof.

We note that the first solution in (62) and the solution in (64) coincide when $y_{5}=\left(1-4 y_{3}^{2}\right) /\left(8 y_{3}\right)$.

4.4. Three Zeros at the Vertices of an Equilateral Triangle. Let $p_{1}, p_{2}$, and $p_{3}$ be at the vertices of an equilateral triangle. Without loss of generality, we can assume that $y_{3}>0$; then, the positions of the zeros are $p_{1}=0, p_{2}=1$, $p_{3}=(1 / 2)+(\sqrt{3} / 2) i, p_{4}=x_{4}+y_{4} i$, and $p_{5}=x_{5}+y_{5} i$.

Since $y_{3} \neq 0$, all the isochronous configurations of this type are given by Theorem 5 . By substituting $\left(x_{3}, y_{3}\right)=$ $((1 / 2),(\sqrt{3} / 2))$ into equation $\tilde{g}_{72}=0$ and solving the resulting equation, we get the following solutions:

$$
\begin{aligned}
& x_{5}=\frac{1}{2}, \\
& y_{5}=-\frac{x_{5}-1}{\sqrt{3}}, \\
& y_{5}=\frac{x_{5}}{\sqrt{3}}, \\
& y_{5}=\frac{1 \pm \sqrt{-12 x_{5}^{2}+12 x_{5}+1}}{2 \sqrt{3}} .
\end{aligned}
$$

The first three solutions provide the following valid solutions of $(8)$ with $\left(x_{4}, y_{4}\right)=\left(x_{40}, y_{40}\right)$ :

$$
\begin{aligned}
& \left(x_{4}, y_{4}\right)=\left(\frac{1}{2}, \frac{2 y_{5}+\sqrt{3}}{4 \sqrt{3} y_{5}-2}\right), \\
& \left(x_{5}, y_{5}\right)=\left(\frac{1}{2}, y_{5}\right), \\
& \left(x_{4}, y_{4}\right)=\left(\frac{x_{5}}{2 x_{5}-1}, \frac{\sqrt{3}\left(x_{5}-1\right)}{6 x_{5}-3}\right), \\
& \left(x_{5}, y_{5}\right)=\left(x_{5},-\frac{x_{5}-1}{\sqrt{3}}\right), \\
& \left(x_{4}, y_{4}\right)=\left(\frac{x_{5}}{2 x_{5}-1}, \frac{x_{5}}{\sqrt{3}\left(2 x_{5}-1\right)}\right), \\
& \left(x_{5}, y_{5}\right)=\left(x_{5}, \frac{x_{5}}{\sqrt{3}}\right) .
\end{aligned}
$$

The last solution provides solutions of (8) satisfying condition $K_{3}$; thus, the solution $\left(x_{4}, y_{4}\right)=\left(x_{40}, y_{40}\right)$ is not defined in this case. Finally, the solutions of (8) with $\left(x_{4}, y_{4}\right)=\left(x_{4}^{(*, 1)}, y_{4}^{(*, 1)}\right)$ and $\left(x_{5}, y_{5}\right)=\left(x_{5}^{(*, 1)}, y_{5}^{\star}\right)$ are not defined when $\left(x_{3}, y_{3}\right)=((1 / 2),(\sqrt{3} / 2))$ because $d=0$.

Analyzing the shape of the solutions of (66), we see that, in the first solution of (66), the zeros $p_{4}$ and $p_{5}$ are on the median of the triangle that passes through the vertex $p_{3}$, in the second solution of (66), the zeros $p_{4}$ and $p_{5}$ are on the median passing through the vertex $p_{2}$, and in the third 
solution, $p_{4}$ and $p_{5}$ are on the median passing through the vertex $p_{1}$. We note that when $y_{5}=-(\sqrt{3} / 2)$, the first solution of (66) is a square of vertices $p_{1}, p_{2}, p_{3}$, and $p_{5}$ and $p_{4}$ at its center.

We have proved the following result.

Lemma 5. Assume that the configuration $\left[p_{1}, \ldots, p_{5}\right]$ is isochronous. The following statements hold.

(a) If $p_{1}, p_{2}$, and $p_{3}$ are at the vertices of an equilateral triangle, then $p_{4}$ and $p_{5}$ are in one of the medians of the triangle, and therefore, $p_{1}, p_{2}, p_{3}, p_{4}$, and $p_{5}$ satisfy statements of Lemma 4.

(b) There exists three different one-parameter families of configurations with three zeros at the vertices of a given equilateral triangle and two zeros in a median of the triangle, one for each median. If the vertices of the equilateral triangle are $p_{1}=0, p_{2}=1$, and $p_{3}=(1 /$ $2)+i(\sqrt{3} / 2)$, then these three one-parameter families of configurations are given by $p_{4}=x_{4}+i y_{4}$ and $p_{5}=$ $x_{5}+i y_{5}$ with $\left(x_{4}, y_{4}\right),\left(x_{5}, y_{5}\right)$ given in (66).

We have plotted the phase portrait of the isochronous vector fields associated to the configurations given by Lemma 5(b) for many values of the variable that acts as a parameter. After analyzing the obtained results, we conjecture the following.

(i) If $\left(x_{4}, y_{4}\right)$ and $\left(x_{5}, y_{5}\right)$ are given by the first solution in (66), then the phase portrait of the isochronous configuration has star topology when $y_{5}<(1 /$ $(2 \sqrt{3}))$ and fork topology when $y_{5}>(1 /(2 \sqrt{3}))$

(ii) If $\left(x_{4}, y_{4}\right)$ and $\left(x_{5}, y_{5}\right)$ are given by the second solution in (66), then the phase portrait of the isochronous configuration has star topology when $x_{5}<(1 / 2)$ and fork topology when $x_{5}>(1 / 2)$

(iii) If $\left(x_{4}, y_{4}\right)$ and $\left(x_{5}, y_{5}\right)$ are given by the third solution in (66), then the phase portrait of the isochronous configuration has fork topology when $x_{5}<(1 / 2)$ and star topology when $x_{5}>(1 / 2)$

4.5. Three Zeros at the Vertices of an Isosceles Triangle. Let $p_{1}, p_{2}$, and $p_{3}$ be at the vertices of an isosceles triangle. Without loss of generality, we can assume that the positions of the zeros are $p_{1}=0, \quad p_{2}=1, \quad p_{3}=(1 / 2)+y_{3} i$, $p_{4}=x_{4}+y_{4} i$, and $p_{5}=x_{5}+y_{5} i$ with $y_{3}>0$.

Since $y_{3} \neq 0$, all the isochronous configurations of this type are given by Theorem 5 . From Theorem 5(d), the solutions of (5) with $\left(x_{4}, y_{4}\right)=\left(x_{4}^{(\star, 1)}, y_{4}^{(\star, 1)}\right)$ and $\left(x_{5}, y_{5}\right)=$ $\left(x_{5}^{(*, 1)}, y_{5}^{\star}\right)$ become

$$
\begin{aligned}
& \left(x_{3}, y_{3}\right)=\left(\frac{1}{2}, y_{3}\right), \\
& \left(x_{4}, y_{4}\right)=\left(\frac{1}{2},-\frac{1}{4 y_{3}}\right), \\
& \left(x_{5}, y_{5}\right)=\left(\frac{1}{2}, \frac{1-4 y_{3}^{2}}{8 y_{3}},\right) .
\end{aligned}
$$

From Theorem 5(c), we obtain the two-parameter family of solutions

$$
\begin{aligned}
& \left(x_{3}, y_{3}\right)=\left(\frac{1}{2}, y_{3}\right), \\
& \left(x_{4}, y_{4}\right)=\left(\frac{1}{2}, \frac{y_{3}+y_{5}}{4 y_{3} y_{5}-1}\right), \\
& \left(x_{5}, y_{5}\right)=\left(\frac{1}{2}, y_{5}\right) .
\end{aligned}
$$

This solution corresponds to the configuration given by Theorem 3(d). We note that the solutions (67) and (68) coincide when

$$
y_{5}=\frac{1-4 y_{3}^{2}}{8 y_{3}} .
$$

From Theorem 5(b), we know the existence of up to six different two-parameter families of solutions with $x_{5} \neq(1 / 2), \quad\left(x_{4}, y_{4}\right)=\left(x_{40}, y_{40}\right)$, and $y_{5}=y_{5}\left(y_{3}, x_{5}\right)$ satisfying $\widetilde{g}_{72}=0$. The exact number of such families will depend on the values of $y_{3}$ and $x_{5}$. Unfortunately, equation $\widetilde{g}_{72}=0$ for the isosceles triangle configurations cannot be solved explicitly. We can solve it numerically by setting the values of the parameters $y_{3}$ and $x_{5}$. Hence, we have proved the following result.

Lemma 6. Assume that $X$ is isochronous and that $p_{1}, p_{2}$, and $p_{3}$ are at the vertices of an isosceles triangle. Let $p_{1}=0$, $p_{2}=1$, and $p_{3}=(1 / 2)+i y_{3}$ with $y_{3}>0$ be the vertices of the isosceles triangle. Then, the following statements hold.

(a) There exist up to six different two-parameter families of isochronous configurations with $x_{5} \neq(1 / 2)$, $\left(x_{4}, y_{4}\right)=\left(x_{40}, y_{40}\right)$, and $y_{5}=y_{5}\left(y_{3}, x_{5}\right)$ satisfying $\tilde{g}_{72}=0$

(b) There exists the two-parameter family given by (68)

(c) There exists the additional one-parameter family given by (67)

We note that configurations given in statements (b) and (c) coincide when

$$
y_{5}=\frac{1-4 y_{3}^{2}}{8 y_{3}} \text {. }
$$

To give some examples of how the families given by Lemma 6 are, now we will analyze numerically the families of solutions of $\widetilde{g}_{72}=0$ with $x_{5} \neq(1 / 2)$ for two particular values of $y_{3}$. We have chosen $y_{3}=(1 / 25)$, which provides a zero near $p_{3}=(1 / 2)$ (i.e., the configuration with $p_{3}$ at the midpoint of the line segment joining $p_{1}$ and $\left.p_{2}\right)$, and $y_{3}=(9 / 10)$, which provides a zero near $p_{3}=(\sqrt{3} / 2)$ (i.e., the configuration with $p_{1}, p_{2}$, and $p_{3}$ at the vertices of an equilateral triangle).

First, fixing the value of $y_{3}=y_{30}$, we find the values of $x_{5}$ where the number of solutions $y_{5}=y_{5}\left(y_{30}, x_{5}\right)$ can change 
by solving numerically the following system of polynomial equations:

$$
\begin{aligned}
\widetilde{g}_{72} & =0, \\
\frac{\mathrm{d} \widetilde{g}_{72}}{\mathrm{~d} y_{5}} & =0 .
\end{aligned}
$$

Let $x_{5}=\alpha_{i}$ for $i=1, \ldots, n$ denote the values of $x_{5}$ corresponding to the solutions of (1), and let $\alpha_{0}=-\infty$ and $\alpha_{n+1}=\infty$. For all $i=0, \ldots, n$, we find the number of solutions of equation $\tilde{g}_{72}=0$ for a value $x_{5} \in\left(\alpha_{i}, \alpha_{i+1}\right)$ by solving numerically the polynomial equation $\widetilde{g}_{72}=0$. We note that due to the symmetry of the configuration, it is sufficient to consider values of $x_{5}<(1 / 2)$. Finally, to understand how the families of solutions are related, we compute numerically the solutions of $\widetilde{g}_{72}=0$ for values of $x_{5}$ near the bifurcation values $\alpha_{i}$ for $i=1, \ldots, n$. Here, we only give the solution at a value $x_{5} \in\left(\alpha_{i}, \alpha_{i+1}\right)$ for all $i=0, \ldots, n$ and at $x_{5}=\alpha_{i}$ for all $i=1, \ldots, n$. These are the results that we have obtained.

Case: $y_{3}=(1 / 25)$

Equation $\tilde{g}_{72}=0$ with $y_{3}=(1 / 25)$ has the following families of solutions.

(i) Two one-parameter families of solutions $y_{5}=y_{5}\left((1 / 25), x_{5}\right)$ when $x_{5} \in\left(\alpha_{0}, \alpha_{1}\right)$ with $\alpha_{0}=$ $-\infty$ and $\alpha_{1}=-0.878799 \ldots$

(ii) Three solutions $y_{5}=y_{5}\left((1 / 25), x_{5}\right)$ when $x_{5}=\alpha_{1}$ (iii) Four one parameter families of solutions $y_{5}=y_{5}$ $\left((1 / 25), x_{5}\right)$ when $x_{5} \in\left(\alpha_{1}, \alpha_{2}\right)$ with $\alpha_{2}=$ $-0.126632 \ldots$

(iv) Five solutions $y_{5}=y_{5}\left((1 / 25), x_{5}\right)$ when $x_{5}=\alpha_{2}$

(v) Six one-parameter families of solutions $y_{5}=y_{5}\left((1 / 25), x_{5}\right)$ when $x_{5} \in\left(\alpha_{2}, \alpha_{3}\right)$ with $\alpha_{3}=0$

(vi) Four one-parameter families of solutions $y_{5}=y_{5}\left((1 / 25), x_{5}\right)$ when $x_{5}=\alpha_{3}$

(vii) Six one-parameter families of solutions $y_{5}=$ $y_{5}\left((1 / 25), x_{5}\right)$ when $x_{5} \in\left(\alpha_{3}, \alpha_{4}\right)$ with $\alpha_{4}=$ $0.0258965 \ldots$

(viii) Five solutions $y_{5}=y_{5}\left((1 / 25), x_{5}\right)$ when $x_{5}=\alpha_{4}$

(ix) Four solutions $y_{5}=y_{5}\left((1 / 25), x_{5}\right)$ when $x_{5} \in\left(\alpha_{4}\right.$, $\left.\alpha_{5}\right)$ with $\alpha_{5}=(1 / 2)$

Now, we give the isochronous configurations $p_{1}=0$, $p_{2}=1, p_{3}=(1 / 2)+i(1 / 25), p_{4}=x_{4}+i y_{4}$, and $p_{5}=x_{5}+$ $i y_{5}$ at a given value $x_{5}$ in each interval $\left(\alpha_{i}, \alpha_{i+1}\right)$ with $i=$ $0, \ldots, 5$ and at $x_{5}=\alpha_{i}$ with $i=1, \ldots, 5$. We will use the following notation: we denote the families of solutions by $f_{i}$ for $i=1, \ldots, 6$, and the notation $f_{i j}$ denotes a solution from which we bifurcate the two families $f_{i}$ and $f_{j}$.

(i) If $x_{5}=-1$, then the two solutions of $\tilde{g}_{72}=0$ provide the isochronous configurations with

$$
\begin{aligned}
& f_{1}:\left(x_{4}, y_{4}\right)=(1.841263521,3.376637013),\left(x_{5}, y_{5}\right)=(-1,-0.1950042462) \\
& f_{2}:\left(x_{4}, y_{4}\right)=(15.61743666,13.23676750),\left(x_{5}, y_{5}\right)=(-1,7.575957509)
\end{aligned}
$$

The phase portrait associated to the isochronous vector field given by $f_{1}$ has the fork topology, and the one given by $f_{2}$ has the star topology. (ii) If $x_{5}=\alpha_{1}$, then the solutions of $\widetilde{g}_{72}=0$ provide the isochronous configurations with

$$
\begin{aligned}
& f_{1}:\left(x_{4}, y_{4}\right)=(1.848444615,3.317980858),\left(x_{5}, y_{5}\right)=\left(\alpha_{1},-0.1654776458\right), \\
& f_{34}:\left(x_{4}, y_{4}\right)=(1.011696918,-0.001515843709),\left(x_{5}, y_{5}\right)=\left(\alpha_{1}, 2.797454186\right), \\
& f_{2}:\left(x_{4}, y_{4}\right)=(16.58023312,13.70216652),\left(x_{5}, y_{5}\right)=\left(\alpha_{1}, 7.491373514\right) .
\end{aligned}
$$


The phase portraits associated to the isochronous vector fields given by $f_{1}$ and $f_{34}$ have the fork topology, and the one given by $f_{2}$ has the star topology. (iii) If $x_{5}=-(1 / 2)$, then the four solutions of $\tilde{g}_{72}=0$ provide the isochronous configurations with

$$
\begin{aligned}
& f_{1}:\left(x_{4}, y_{4}\right)=(1.867414547,3.115470806),\left(x_{5}, y_{5}\right)=\left(-\frac{1}{2},-0.08264979077\right), \\
& f_{3}:\left(x_{4}, y_{4}\right)=(-0.1232221041,-0.05229100159),\left(x_{5}, y_{5}\right)=\left(-\frac{1}{2}, 1.064778767\right), \\
& f_{4}:\left(x_{4}, y_{4}\right)=(6.907958078,-2.048938409),\left(x_{5}, y_{5}\right)=\left(-\frac{1}{2}, 4.644413617\right), \\
& f_{2}:\left(x_{4}, y_{4}\right)=(20.94262385,15.91785952),\left(x_{5}, y_{5}\right)=\left(-\frac{1}{2}, 7.206590362\right) .
\end{aligned}
$$

Note that, from the family $f_{34}$ given in (ii), bifurcate two families of solutions that we denote by $f_{3}$ and $f_{4}$.

The phase portraits associated to the isochronous vector fields given by $f_{1}, f_{3}$, and $f_{4}$ have the fork topology, and the one given by $f_{2}$ has the star topology.

(iv) If $x_{5}=\alpha_{2}$, then the five solutions of $\widetilde{g}_{72}=0$ provide the isochronous configurations with

$$
\begin{aligned}
& f_{56}:\left(x_{4}, y_{4}\right)=(-0.4495265553,0.9614485424),\left(x_{5}, y_{5}\right)=\left(\alpha_{2},-0.05979381213\right), \\
& f_{1}:\left(x_{4}, y_{4}\right)=(1.878034978,2.879573610),\left(x_{5}, y_{5}\right)=\left(\alpha_{2},-0.01757256381\right), \\
& f_{3}:\left(x_{4}, y_{4}\right)=(-0.03560001187,-0.03645028451),\left(x_{5}, y_{5}\right)=\left(\alpha_{2}, 0.4152272923\right), \\
& f_{4}:\left(x_{4}, y_{4}\right)=(16.27721421,-6.804796775),\left(x_{5}, y_{5}\right)=\left(\alpha_{2}, 5.385171289\right), \\
& f_{2}:\left(x_{4}, y_{4}\right)=(30.02386019,20.82877636),\left(x_{5}, y_{5}\right)=\left(\alpha_{2}, 6.890094940\right) .
\end{aligned}
$$

The phase portraits associated to the isochronous vector fields given by $f_{1}, f_{3}, f_{4}$, and $f_{56}$ have the fork topology, and the one given by $f_{2}$ has the star topology. (v) If $x_{5}=-(1 / 20)$, then the six solutions of $\tilde{g}_{72}=0$ provide the isochronous configurations with

$$
\begin{aligned}
& f_{5}:\left(x_{4}, y_{4}\right)=(-0.1579167010,0.4639398830),\left(x_{5}, y_{5}\right)=\left(-\frac{1}{20},-0.04620281861\right), \\
& f_{6}:\left(x_{4}, y_{4}\right)=(-0.6577053363,1.452342544),\left(x_{5}, y_{5}\right)=\left(-\frac{1}{20},-0.01438561542\right), \\
& f_{1}:\left(x_{4}, y_{4}\right)=(1.878710630,2.825402973),\left(x_{5}, y_{5}\right)=\left(-\frac{1}{20},-0.006635633004\right), \\
& f_{3}:\left(x_{4}, y_{4}\right)=(0.001439481676,0.002093770845),\left(x_{5}, y_{5}\right)=\left(-\frac{1}{20}, 0.2910582405\right), \\
& f_{4}:\left(x_{4}, y_{4}\right)=(19.64404887,-8.633711360),\left(x_{5}, y_{5}\right)=\left(-\frac{1}{20}, 5.509564199\right), \\
& f_{2}:\left(x_{4}, y_{4}\right)=(33.35628670,22.67993219),\left(x_{5}, y_{5}\right)=\left(-\frac{1}{20}, 6.819934961\right) .
\end{aligned}
$$

The phase portraits associated to the isochronous vector fields given by $f_{1}, f_{3}, f_{4}, f_{5}$, and $f_{6}$ have the fork topology, and the one given by $f_{2}$ has the star topology. 
(vi) If $x_{5}=0$, then the four solutions of $\widetilde{g}_{72}=0$ provide the three isochronous configurations with

$$
\begin{aligned}
& f_{3}:\left(x_{4}, y_{4}\right)=(0.02289908105,0.05342007123),\left(x_{5}, y_{5}\right)=(0,0.1930349677), \\
& f_{4}:\left(x_{4}, y_{4}\right)=(22.37646822,-10.13881729),\left(x_{5}, y_{5}\right)=(0,5.587207040), \\
& f_{2}:\left(x_{4}, y_{4}\right)=(36.06938270,24.19789721),\left(x_{5}, y_{5}\right)=(0,6.773091325) .
\end{aligned}
$$

The fourth solution of $\widetilde{g}_{72}=0$ is $\left(x_{5}, y_{5}\right)=(0,0)$, and it does not provide an isochronous configuration. At this solution, the three families of solutions $\quad y_{5}=y_{5}\left((1 / 25), x_{5}\right) \quad$ of $\quad \tilde{g}_{72}=0$ corresponding to $f_{5}, f_{6}$, and $f_{1}$ coincide.
The phase portraits associated to the isochronous vector fields given by $f_{3}$ and $f_{4}$ have the fork topology, and the one given by $f_{2}$ has the star topology.

(vii) If $x_{5}=(1 / 100)$, then the six solutions of $\widetilde{g}_{72}=0$ provide the isochronous configurations with

$$
\begin{aligned}
& f_{5}:\left(x_{4}, y_{4}\right)=(-0.7105285631,1.616886803),\left(x_{5}, y_{5}\right)=\left(\frac{1}{100}, 0.002419518544\right), \\
& f_{6}:\left(x_{4}, y_{4}\right)=(-0.03179981882,0.2585482764),\left(x_{5}, y_{5}\right)=\left(\frac{1}{100}, 0.01652281623\right), \\
& f_{1}:\left(x_{4}, y_{4}\right)=(1.878769832,2.781350016),\left(x_{5}, y_{5}\right)=\left(\frac{1}{100}, 0.001277977173\right), \\
& f_{3}:\left(x_{4}, y_{4}\right)=(0.02541034717,0.07171752269),\left(x_{5}, y_{5}\right)=\left(\frac{1}{100}, 0.1670678630\right) \\
& f_{4}:\left(x_{4}, y_{4}\right)=(22.98795700,-10.47759276),\left(x_{5}, y_{5}\right)=\left(\frac{1}{100}, 5.602426971\right) \\
& f_{2}:\left(x_{4}, y_{4}\right)=(36.67728304,24.53909014),\left(x_{5}, y_{5}\right)=\left(\frac{1}{100}, 6.763618187\right) .
\end{aligned}
$$

The phase portraits associated to the isochronous vector fields given by $f_{1}, f_{3}, f_{4}, f_{5}$, and $f_{6}$ have the fork topology, and the one given by $f_{2}$ has the star topology. (viii) If $x_{5}=\alpha_{4}$, then the five solutions of $\widetilde{g}_{72}=0$ provide the isochronous configurations with

$$
\begin{aligned}
& f_{5}:\left(x_{4}, y_{4}\right)=(-0.7213143913,1.653877238),\left(x_{5}, y_{5}\right)=\left(\alpha_{4}, 0.006016399475\right), \\
& f_{36}:\left(x_{4}, y_{4}\right)=(0.01527596548,0.1507327875),\left(x_{5}, y_{5}\right)=\left(\alpha_{4}, 0.08456876555\right), \\
& f_{1}:\left(x_{4}, y_{4}\right)=(1.878709929,2.769422027),\left(x_{5}, y_{5}\right)=\left(\alpha_{4}, 0.003275093401\right), \\
& f_{4}:\left(x_{4}, y_{4}\right)=(24.01178345,-11.04616649),\left(x_{5}, y_{5}\right)=\left(\alpha_{4}, 5.626418095\right), \\
& f_{2}:\left(x_{4}, y_{4}\right)=(37.69558803,25.11140165),\left(x_{5}, y_{5}\right)=\left(\alpha_{4}, 6.748486215\right) .
\end{aligned}
$$

The phase portraits associated to the isochronous vector fields given by $f_{1}, f_{36}, f_{4}$, and $f_{5}$ have the fork topology, and the one given by $f_{2}$ has the star topology. 
(ix) If $x_{5}=(3 / 10)$, then the four solutions of $\tilde{g}_{72}=0$ provide the isochronous configurations with

$$
\begin{aligned}
& f_{5}:\left(x_{4}, y_{4}\right)=(-0.8239444869,2.112991297),\left(x_{5}, y_{5}\right)=\left(\frac{3}{10}, 0.03808849818\right), \\
& f_{1}:\left(x_{4}, y_{4}\right)=(1.871423616,2.543910571),\left(x_{5}, y_{5}\right)=\left(\frac{3}{10}, 0.03043761676\right), \\
& f_{4}:\left(x_{4}, y_{4}\right)=(66.70297000,-35.33061762),\left(x_{5}, y_{5}\right)=\left(\frac{3}{10}, 6.006030328\right), \\
& f_{2}:\left(x_{4}, y_{4}\right)=(80.32320661,49.44048347),\left(x_{5}, y_{5}\right)=\left(\frac{3}{10}, 6.472332652\right) .
\end{aligned}
$$

The phase portraits associated to the isochronous vector fields given by $f_{1}, f_{4}$, and $f_{5}$ have the fork topology, and the one given by $f_{2}$ has the star topology.

We note that when $x_{5} \longrightarrow(1 / 2)$, the solution families $f_{2}$ and $f_{4}$ tend to a solution of $\tilde{g}_{72}=0$ with $\left(x_{5}, y_{5}\right)=((1 / 2),(25 / 4))$. This solution does not provide a solution of (8) because $D_{1}^{2}+D_{2}^{2}=0$, and therefore, $\left(x_{40}, y_{40}\right)$ is not defined. Moreover, $\left(x_{4}, y_{4}\right) \longrightarrow(+\infty,+\infty)$ along the solutions' families $f_{2}$ and $f_{4}$ when $x_{5} \longrightarrow(1 / 2)$. On the contrary, the solution families $f_{1}$ and $f_{5}$ tend to a solution of $\tilde{g}_{72}=0$ with $\left(x_{5}, y_{5}\right)=((1 / 2),(1 / 25))$. This solution does not provide a valid solution because $p_{3}=p_{5}$. In this case, $\left(x_{4}, y_{4}\right) \longrightarrow(1.855487879,2.348621176)$ and $\left(x_{4}, y_{4}\right) \longrightarrow(-0.8554878791,2.348621176)$ along the solutions' families $f_{1}$ and $f_{5}$, respectively, when $x_{5} \longrightarrow(1 / 2)$.

Case: $y_{3}=(9 / 10)$ :

Equation $\tilde{g}_{72}=0$ with $y_{3}=(9 / 10)$ has the following families of solutions.

(i) Two one-parameter families of solutions $y_{5}=y_{5}\left((9 / 10), x_{5}\right) \quad$ when $\quad x_{5} \in\left(\alpha_{0}, \alpha_{1}\right) \quad$ with $\alpha_{0}=-\infty$ and $\alpha_{1}=-0.0137965 \ldots$ (ii) Three solutions $y_{5}=y_{5}\left((9 / 10), x_{5}\right)$ when $x_{5}=\alpha_{1}$

(iii) Four one-parameter families of solutions $y_{5}=y_{5}\left((9 / 10), x_{5}\right)$ when $x_{5} \in\left(\alpha_{1}, \alpha_{2}\right)$ with $\alpha_{2}=0$

(iv) Two solutions $y_{5}=y_{5}\left((9 / 10), x_{5}\right)$ when $x_{5}=\alpha_{2}$

(v) Four one-parameter families of solutions $y_{5}=y_{5}\left((9 / 10), x_{5}\right) \quad$ when $\quad x_{5} \in\left(\alpha_{2}, \alpha_{3}\right) \quad$ with $\alpha_{3}=(1 / 2)$

Now we give the isochronous configurations $p_{1}=0$, $p_{2}=1, p_{3}=(1 / 2)+i(9 / 10), p_{4}=x_{4}+i y_{4}$, and $p_{5}=x_{5}+$ $i y_{5}$ at a given value $x_{5}$ in each interval $\left(\alpha_{i}, \alpha_{i+1}\right)$ with $i=$ $0, \ldots, 3$ and at $x_{5}=\alpha_{i}$ with $i=1,2$, and 3 . As above, $f_{i}$ with $i=1, \ldots, 4$ denotes the families of solutions and $f_{i j}$ denotes a solution from which we bifurcate the two families $f_{i}$ and $f_{j}$.

(i) If $x_{5}=-(1 / 2)$, then two solutions of $\widetilde{g}_{72}=0$ provide the isochronous configurations with

$$
\begin{aligned}
& f_{1}:\left(x_{4}, y_{4}\right)=(0.2273153488,0.1290590866),\left(x_{5}, y_{5}\right)=\left(-\frac{1}{2},-0.2873400074\right), \\
& f_{2}:\left(x_{4}, y_{4}\right)=(0.2050985302,0.3874083254),\left(x_{5}, y_{5}\right)=\left(-\frac{1}{2}, 0.8291856821\right) .
\end{aligned}
$$

The phase portrait associated to the isochronous vector field given by $f_{1}$ has the fork topology, and the one given by $f_{2}$ has the star topology. (ii) If $x_{5}=\alpha_{1}$, then the solutions of $\widetilde{g}_{72}=0$ provide the isochronous configurations with

$$
\begin{aligned}
f_{1}:\left(x_{4}, y_{4}\right) & =(-0.03026264711,-0.01730520403),\left(x_{5}, y_{5}\right)=\left(\alpha_{1},-0.007886903324\right), \\
f_{2}:\left(x_{4}, y_{4}\right) & =(0.06989971864,-0.1553809110),\left(x_{5}, y_{5}\right)=\left(\alpha_{1}, 0.01504460688\right), \\
f_{34}:\left(x_{4}, y_{4}\right) & =(-0.1760156610,0.2985515049),\left(x_{5}, y_{5}\right)=\left(\alpha_{1}, 0.1098723790\right) .
\end{aligned}
$$


The phase portrait associated to the isochronous vector field given by $f_{1}$ has the fork topology, and the ones given by $f_{2}$ and $f_{34}$ have the star topology. (iii) If $x_{5}=-(1 / 100)$, then the four isochronous configurations are given by

$$
\begin{aligned}
& f_{1}:\left(x_{4}, y_{4}\right)=(-0.03421033191,-0.01956401179),\left(x_{5}, y_{5}\right)=\left(-\frac{1}{100},-0.005716181684\right), \\
& f_{2}:\left(x_{4}, y_{4}\right)=(0.07267869959,-0.1594371587),\left(x_{5}, y_{5}\right)=\left(-\frac{1}{100}, 0.01081966297\right) \\
& f_{3}:\left(x_{4}, y_{4}\right)=(-0.1515620634,0.2353327280),\left(x_{5}, y_{5}\right)=\left(-\frac{1}{100}, 0.05238535873\right) \\
& f_{4}:\left(x_{4}, y_{4}\right)=(-0.1965428001,0.3621824381),\left(x_{5}, y_{5}\right)=\left(-\frac{1}{100}, 0.1660213488\right)
\end{aligned}
$$

Note that, from the family $f_{34}$ given in (ii), bifurcate two families of solutions that we denote by $f_{3}$ and $f_{4}$.

The phase portrait associated to the isochronous vector field given by $f_{1}$ has the fork topology, and the ones given by $f_{2}, f_{3}$, and $f_{4}$ have the star topology.

(iv) If $x_{5}=0$, then the two solutions of $\widetilde{g}_{72}=0$ provide a unique isochronous configuration with

$$
f_{4}:\left(x_{4}, y_{4}\right)=(-0.2129653240,0.4198478686),\left(x_{5}, y_{5}\right)=(0,0.2147580631)
$$

The other solution of $\tilde{g}_{72}=0$ is $\left(x_{5}, y_{5}\right)=(0,0)$, and it does not provide isochronous configurations. At this solution, the three families of solutions $y_{5}=$ $y_{5}\left((9 / 10), x_{5}\right)$ of $\widetilde{g}_{72}=0$ corresponding to $f_{1}, f_{2}$, and $f_{3}$ coincide.
The phase portrait associated to the isochronous vector field given by $f_{4}$ has the star topology.

(v) If $x_{5}=(3 / 10)$, then the four solutions of $\widetilde{g}_{72}=0$ provide a unique isochronous configuration with

$$
\begin{aligned}
& f_{3}:\left(x_{4}, y_{4}\right)=(0.2692037018,-0.1777437971),\left(x_{5}, y_{5}\right)=\left(\frac{3}{10},-0.3420260454\right), \\
& f_{2}:\left(x_{4}, y_{4}\right)=(0.3264300730,-0.3521082462),\left(x_{5}, y_{5}\right)=\left(\frac{3}{10},-0.1880843834\right), \\
& f_{1}:\left(x_{4}, y_{4}\right)=(-0.8646324249,-0.4976775204),\left(x_{5}, y_{5}\right)=\left(\frac{3}{10}, 0.1697279313\right), \\
& f_{4}:\left(x_{4}, y_{4}\right)=(-0.9156482786,1.092880633),\left(x_{5}, y_{5}\right)=\left(\frac{3}{10}, 0.3673099827\right) .
\end{aligned}
$$

The phase portrait associated to the isochronous vector field given by $f_{1}$ has the fork topology, and the ones given by $f_{2}, f_{3}$, and $f_{4}$ have the star topology.

We note that when $x_{5} \longrightarrow(1 / 2)$, the solution families $f_{1}$ and $f_{4}$ tend to a solution of $\tilde{g}_{72}=0$ with $\left(x_{5}, y_{5}\right)=((1 / 2),(5 / 18))$. This solution does not provide a solution of (8) because $D_{1}^{2}+D_{2}^{2}=0$, and therefore, $\left(x_{40}, y_{40}\right)$ is not defined. Moreover, $\left(x_{4}, y_{4}\right) \longrightarrow(0,0)$ along the solutions' families $f_{1}$ and $f_{4}$ when $x_{5} \longrightarrow(1 / 2)$. The solution families $f_{2}$ and $f_{3}$ tend to the following solutions, which provide isochronous configurations: 


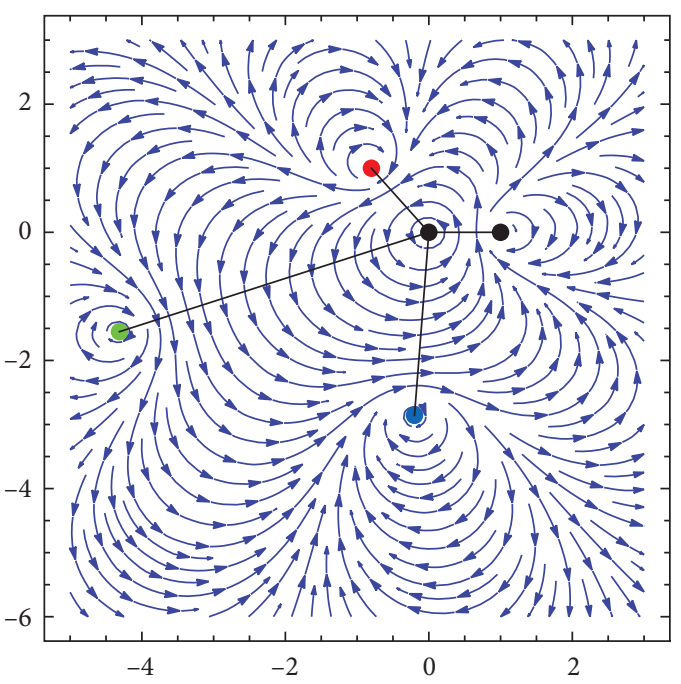

(a)

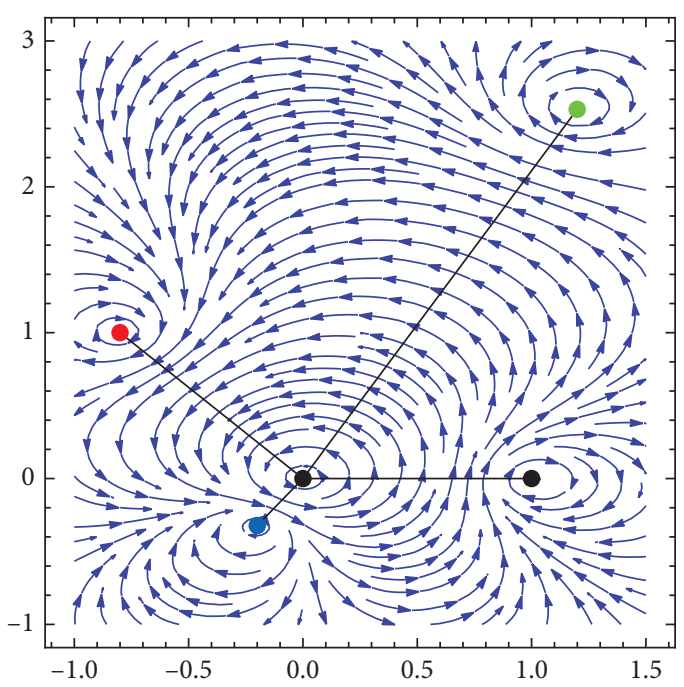

(c)

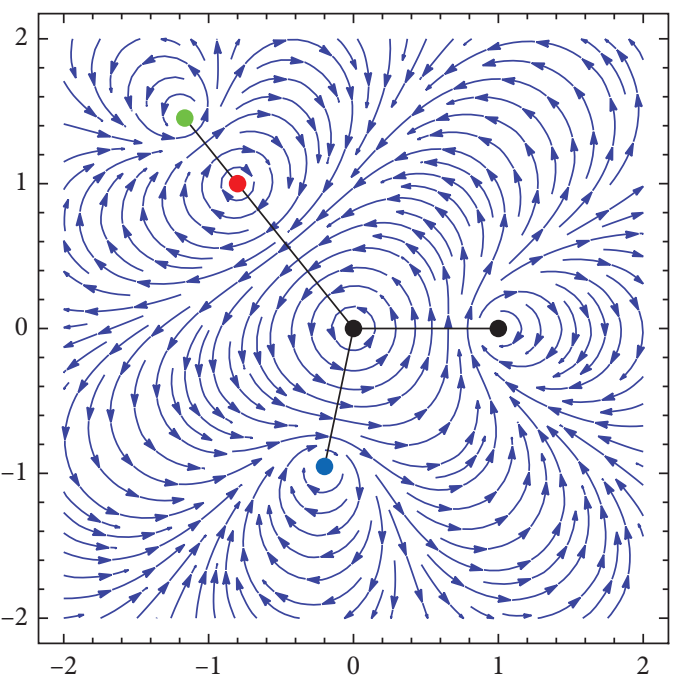

(b)

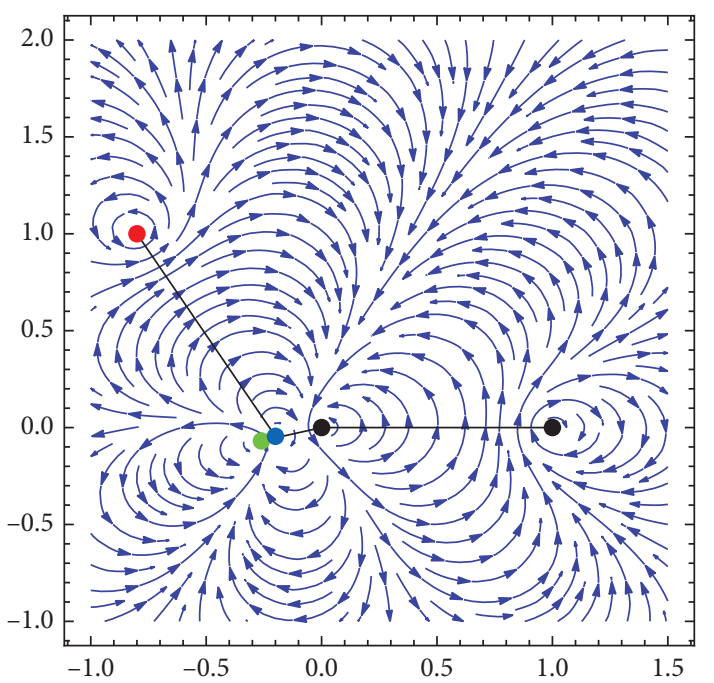

(d)

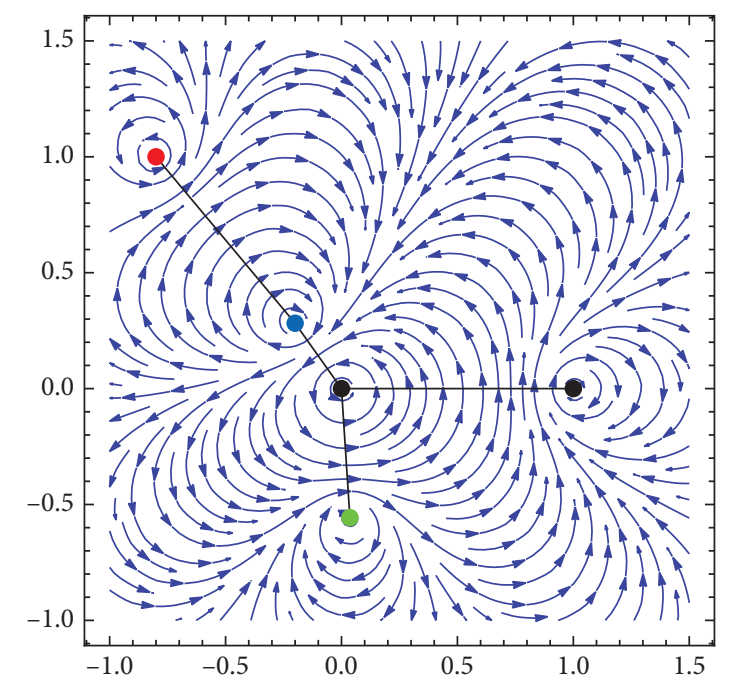

(e)

FIGURE 2: The isochronous configurations with $\left(x_{3}, y_{3}, x_{5}\right)=(-(8 / 10), 1,-(2 / 10))$. (a) $\left(x_{4}, y_{4}, y_{5}\right) \approx(-4.312,-1.551,-2.856)$. (b) $\left(x_{4}, y_{4}, y_{5}\right) \approx(-1.164,1.452,-0.952)$. $\left(x_{4}, y_{4}, y_{5}\right) \approx(0.036,-0.556,0.282)$.

(c) $\left(x_{4}, y_{4}, y_{5}\right) \approx(1.199,2.531,-0.322)$. $\left(x_{4}, y_{4}, y_{5}\right) \approx(-0.260,0.069,-0.045)$. 


$$
\begin{aligned}
& f_{3}:\left(x_{4}, y_{4}\right)=\left(\frac{1}{2},-0.2183503419\right),\left(x_{5}, y_{5}\right)=\left(\frac{1}{2},-0.3816496581\right), \\
& f_{2}:\left(x_{4}, y_{4}\right)=\left(\frac{1}{2},-0.3816496581\right),\left(x_{5}, y_{5}\right)=\left(\frac{1}{2},-0.2183503419\right) .
\end{aligned}
$$

The phase portraits associated to the isochronous vector fields given by $f_{2}$ and $f_{3}$ have the star topology.

4.6. More Examples. In the previous sections, we have studied particular cases where the configuration of some of the zeros is symmetric. By proceeding as in the last section, we could analyze the isochronous configurations when $p_{1}$, $p_{2}$, and $p_{3}$ are in an arbitrary triangle obtaining in this way isochronous configurations having no symmetries. For example, if $x_{3}=-(8 / 10), y_{3}=1$, and $x_{5}=-(2 / 10)$, then we obtain five isochronous configurations that are given by

$$
\begin{aligned}
& \left(x_{4}, y_{4}\right)=(-4.312509774,-1.551253710),\left(x_{5}, y_{5}\right)=\left(-\frac{2}{10},-2.856970625\right), \\
& \left(x_{4}, y_{4}\right)=(-1.164499645,1.452969805),\left(x_{5}, y_{5}\right)=\left(-\frac{2}{10},-0.9524134598\right), \\
& \left(x_{4}, y_{4}\right)=(1.199397231,2.531219547),\left(x_{5}, y_{5}\right)=\left(-\frac{2}{10},-0.3220182178\right), \\
& \left(x_{4}, y_{4}\right)=(-0.2605712264,-0.06904887457),\left(x_{5}, y_{5}\right)=\left(-\frac{2}{10},-0.04520614246\right), \\
& \left(x_{4}, y_{4}\right)=(0.03635581518,-0.5567650011),\left(x_{5}, y_{5}\right)=\left(-\frac{2}{10}, 0.2820385942\right) .
\end{aligned}
$$

In Figure 2, we show the phase portraits of the isochronous vector fields associated to these configurations with their corresponding 5-trees. We observe that the phase portrait of the first and the third configurations have the star topology, while the other three have the fork topology. Note that, in the second configuration, three zeros are in the bisector line of the other two, and this does not happen in the fourth and the fifth.

For the moment, we have not been able to find examples of isochronous vector fields having a phase portrait with line topology whose zeros are not aligned. We conjecture that there are no isochronous vector fields having a phase portrait with line topology whose zeros are not aligned.

\section{Conflicts of Interest}

The authors declare that they have no conflicts of interest.

\section{Acknowledgments}

This work was partially supported by Tecnologico de Monterrey. The second author was partially supported by the Ministerio de Ciencia, Innovación y Universidades, Agencia Estatal de Investigación grants MTM2016-77278-P (FEDER) and PID2019-104658GB-I00 (FEDER).

\section{References}

[1] M.-E. Frías-Armenta and J. Muciño-Raymundo, "Topological and analytical classification of vector fields with only isochronous centres," Journal of Difference Equations and Applications, vol. 19, no. 10, pp. 1694-1728, 2013.

[2] F. Klein, On Riemann's Theory of Algebraic Functions and Their Integrals, Dover, New York, NY, USA, 1963.

[3] J. Muciño-Raymundo, "Complex structures adapted to smooth vector fields," Mathematische Annalen, vol. 322, no. 2, pp. 229-265, 2002.

[4] M. J. Álvarez, A. Gasull, and R. Prohens, "Configurations of critical points in complex polynomial differential equations," Nonlinear Analysis: Theory, Methods \& Applications, vol. 71, no. 3-4, pp. 923-934, 2009.

[5] B. Branner and K. Dias, "Classification of complex polynomial vector fields in one complex variable," Journal of Difference Equations and Applications, vol. 16, no. 5-6, pp. 463-517, 2010.

[6] J. Reyn, Phase Portraits of Planar Quadratic Systems, Srpinger, New York, NY, USA, 2007.

[7] K. Dias and L. Tan, "On parameter space of complex polynomial vector fields in C," Journal of Differential Equations, vol. 260, no. 1, pp. 628-652, 2016.

[8] K. Dias and A. Garijo, "On the separatrix graph of a rational vector field on the Riemann sphere," Journal of Differential Equations, vol. 282, pp. 541-565, 2021.

[9] A. Alvarez-Parrilla and J. Muciño-Raymundo, "Dynamics of singular complex analytic vector fields with essential singularities I," Conformal Geometry and Dynamics of the American Mathematical Society, vol. 21, pp. 126-224, 2017.

[10] A. Alvarez-Parrilla and J. Muciño-Raymundo, "Dynamics of singular complex analytic vector fields with essential singularities II," 2019, https://arxiv.org/abs/1906.04207.

[11] M. Marden, "Geometry of polynomials," American Mathematical Society, vol. 3, 1966. 
[12] S. Lang, Algebra, Addison-Wesley, Boston, MA, USA, 3rd edition, 1993.

[13] P. Olver, "Classical invariant theory," London Math. Soc. Student Texts, Cambridge University Press, New York, NY, USA, 1999. 